\title{
A topology optimization method in rarefied gas flow problems using the Boltzmann equation
}

\section{$\operatorname{AUTHOR}(S)$ :}

Sato, A.; Yamada, T.; Izui, K.; Nishiwaki, S.; Takata, S.

\section{CITATION:}

Sato, A. ... [et al]. A topology optimization method in rarefied gas flow problems using the Boltzmann equation. Journal of Computational Physics 2019, 395: 60-84

\section{ISSUE DATE:}

2019-10-15

URL:

http://hdl.handle.net/2433/243216

\section{RIGHT:}

(c) 2019. This manuscript version is made available under the CC-BY-NC-ND 4.0 license

http://creativecommons.org/licenses/by-nc-nd/4.0/:; The full-text file will be made open to the public on 15 October 2021 in accordance with publisher's 'Terms and Conditions for Self-Archiving'.; この論文は出版社版でありません。引 用の際には出版社版をご確認ご利用ください。; This is not the published version. Please cite only the published version. 


\title{
A topology optimization method in rarefied gas flow problems using the Boltzmann equation
}

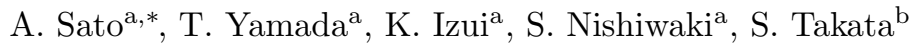 \\ ${ }^{a}$ Department of Mechanical Engineering and Science, Kyoto University, Kyoto 615-8540, Japan. \\ ${ }^{b}$ Department of Aeronautics and Astronautics, Kyoto University, Kyoto 615-8540, Japan.
}

\begin{abstract}
This paper presents a topology optimization method in rarefied gas flow problems to obtain the optimal structure of a flow channel as a configuration of gas and solid domains. In this paper, the kinetic equation, the governing equation of rarefied gas flows, is extended over the entire design domain including solid domains assuming the solid as an imaginary gas for implicitly handling the gas-solid interfaces in the optimization process. Based on the extended equation, a 2D flow channel design problem is formulated, and the design sensitivity is obtained based on the Lagrange multiplier method and adjoint variable method. Both the rarefied gas flow and the adjoint flow are computed by a deterministic method based on a finite discretization of the molecular velocity space, rather than the DSMC method. The validity and effectiveness of our proposed method are confirmed through several numerical examples.
\end{abstract}

Keywords: Topology Optimization, Rarefied Gas Flow, Boltzmann Equation, BGK Equation, Sensitivity Analysis

\section{Introduction}

A rarefied gas is a gas whose mean free path is not negligible compared to the scale of the flow like a lowpressure gas or gas in small systems. The behavior of rarefied gas flows cannot be correctly described by the Navier-Stokes (NS) equation. It should be described by the Boltzmann equation, based on the molecular gas

5 dynamics. Rarefied gas flows have a unique property that steady flows can be induced by thermal gradients even without external forces. There are several devices utilizing this property, including the Knudsen pump proposed by Knudsen in 1910. The Knudsen pump needs no moving parts since its driving force is thermal gradients. From this advantage and the recent improvement of microfabrication techniques, the pump is expected to be applied as a micro-pump [I, [2]. It is also indicated that the Knudsen pump can be used as 10 a gas separator [3] because of the difference of the transport rate of each gas species in rarefied gas regime. Besides, the force produced by a thermal gradient in a rarefied gas can be used in sensors [4] and actuators [5].

However, the structures of these devices using rarefied gas flows are limited to relatively simple structures and do not necessarily provide their maximum performance. One possible approach to tackle this problem 15 is the introduction of structural optimization methods. Structural optimization enables us to design devices based on mathematical and physical principles, which ensures the improvement of the target performance and leads to the reduction of the design period.

Structural optimization methods are usually categorized into three types: sizing $[\mathbf{6}, \mathbf{G}]$, shape $[\mathbf{8}, \mathbf{g}]$ and topology optimization [10, Ш]]. Among them, topology optimization is known to have the highest degree of design freedom because it allows structures to change not only the shape of their boundaries but also

\footnotetext{
* Corresponding author

Email address: satou.ayami.34u@kyoto-u.jp (A. Sato )
} 
the number of inner holes. In topology optimization, a structural optimization problem is replaced with a material distribution problem, where a structure is expressed as a distribution of the presence or absence of the material in a design domain. Bendsøe and Kikuchi first presented the homogenization design method in order to solve this material distribution problem in topology optimization [10]. Density methods [12, 1.3] are another widely used topology optimization method, where a normalized density is considered as a design variable. In addition, level set-based approaches [14, 15, [6] have also attracted attention.

Topology optimization developed mainly for structural mechanics problems in its beginning, for example,

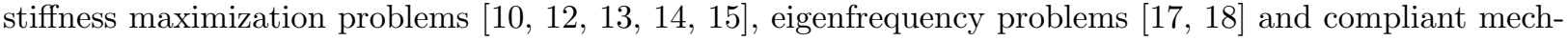
anism problems [16, 1.9, 20]. It has since been applied to other physics problems, such as thermal problems $[21,[22]$, electromagnetic wave problems [2:3] and fluid dynamics problems.

In the fluid dynamics field, Borrvall and Peterssom pioneered topology optimization for Stokes flows based on the density method [24]. They succeeded in obtaining optimal channel structures for minimization problems of energy dissipation. In particular, the whole design domain is considered to be composed of a porous medium, and fluid and solid domains are represented as the parts with high and low permeability,

35 respectively. This method makes it possible that no-slip boundary conditions are implicitly satisfied on the fluid-solid interfaces. Their work was then extended for steady-state incompressible NS flows [25, [26] and unsteady incompressible NS flows [27]. In addition, topology optimization has been applied to developments of fluidic devices [205, [28, [29, 30]. Furthermore, topology optimization methods for multiphysics problems, such as fluid-structure [3]], fluid-electric [32], fluid-thermal [33] and electric-fluid-thermal-structure problems

40 [34], have been also proposed. As is the case of density methods, level set-based approaches have been applied to topology optimization in fluid dynamics problems [35, 36, 37, 38].

While the above studies used the finite element method (FEM) for the analysis of flow fields, there are also studies using other analysis methods, such as the finite volume method (FVM) [3.] and the lattice Boltzmann method (LBM). LBM is an analysis method to obtain the solution of the NS equations by solving

45 a partial difference equation similar to the Boltzmann equation. It is getting more popular because of its suitability for parallel computing and no need of analyzing the Poisson equation for pressure [41], 41, 42]. In topology optimization methods using LBM, several approaches have been proposed to approximate the no-slip boundary condition on the fluid-solid interfaces. The popular approach is the introduction of the porosity model, implemented by the replacement of the flow velocity with the one depending on the design

${ }_{50}$ variable [4.3, 44, 45, 46] or the addition of the fictitious force [47]. Eaniewski-Wołłk and Rokicki presented the method compatible with the multiple GPU computation, based on this approach [48]. As another approach, Nørgaard et al. used the partial bounce-back model [4.9]. However, all of the above studies, even ones using LBM, target only fluid whose mean free path is negligibly small as compared to the system length.

Therefore, a topology optimization method in rarefied gas flow problems is proposed and applied to design problems of a 2-D flow channel. In this method, the gas-solid interfaces, namely the wall of the channel, are implicitly handled through the extension of the kinetic equation in analogy with topology optimization using LBM based on the porosity model. The derivation of the design sensitivity based on the extended equation have also been presented in those previous studies. To follow the procedures of computing the design sensitivity in those, a deterministic numerical method based on the discretized molecular velocity space in rarefied gas flow analyses is adopted, rather than the Direct Simulation Monte Carlo (DSMC) method [50], a well-known stochastic method.

In the following sections, the Boltzmann equation and its model equation are briefly described at first. Then, the topology optimization method we use in this study is outlined. Next, the optimization problem is formulated and the numerical implementation is described. Finally, through several numerical examples, the validity and usefulness of our proposed method are confirmed.

\section{Boltzmann equation}

The Boltzmann equation describes the behavior of velocity distribution function $\hat{f}(\hat{t}, \hat{\boldsymbol{x}}, \hat{\boldsymbol{\zeta}})$ as below:

$$
\frac{\partial \hat{f}}{\partial \hat{t}}+\hat{\zeta} \cdot \hat{\nabla} \hat{f}=C(\hat{f})
$$


where $\hat{t} \in \mathbb{R}^{+}$is the time, $\hat{\boldsymbol{x}} \in \mathbb{R}^{3}$ is position vector, $\hat{\boldsymbol{\zeta}} \in \mathbb{R}^{3}$ is molecular velocity vector and $C(\hat{f})$ denotes the collision term. Since the collision term incurs a severe computational cost, several kinetic equations that simplify the collision term have been proposed. Among them, the following Bhatnagar-Gross-Krook (BGK) equation [5]] is widely used:

$$
\begin{aligned}
& \frac{\partial \hat{f}}{\partial \hat{t}}+\hat{\boldsymbol{\zeta}} \cdot \hat{\nabla} \hat{f}=-\frac{1}{\hat{\tau}}\left(\hat{f}-\hat{f}^{\mathrm{eq}}(\hat{\rho}, \hat{\boldsymbol{u}}, \hat{T})\right), \\
& \hat{f}^{\mathrm{eq}}(\hat{\rho}, \hat{\boldsymbol{u}}, \hat{T})=\frac{\hat{\rho}}{(2 \pi R \hat{T})^{3 / 2}} \exp \left(-\frac{\|\hat{\boldsymbol{\zeta}}-\hat{\boldsymbol{u}}\|^{2}}{2 R \hat{T}}\right),
\end{aligned}
$$

where $\hat{\tau}$ is the mean free time, $R$ is the gas constant and $\hat{f}^{\text {eq }}$ is the local equilibrium distribution function. The macroscopic physical quantities such as density $\hat{\rho}$, flow velocity $\hat{\boldsymbol{u}}$ and temperature $\hat{T}$ are calculated as moments of the velocity distribution function:

$$
\hat{\rho}=\int_{\mathbb{R}^{3}} \hat{f} d \hat{\boldsymbol{\zeta}}, \quad \hat{\boldsymbol{u}}=\frac{1}{\hat{\rho}} \int_{\mathbb{R}^{3}} \hat{\boldsymbol{\zeta}} \hat{f} d \hat{\boldsymbol{\zeta}}, \quad \hat{T}=\frac{1}{3 R \hat{\rho}} \int_{\mathbb{R}^{3}}\|\hat{\boldsymbol{\zeta}}-\hat{\boldsymbol{u}}\|^{2} \hat{f} d \hat{\boldsymbol{\zeta}}
$$

In this study, the BGK equation is made dimensionless as follows:

$$
\begin{aligned}
& \operatorname{Sh} \frac{\partial f}{\partial t}+\boldsymbol{\zeta} \cdot \nabla f=-\frac{2}{\sqrt{\pi}} \frac{\rho}{\mathrm{Kn}}\left(f-f^{\mathrm{eq}}(\rho, \boldsymbol{u}, T)\right), \\
& f^{\mathrm{eq}}(\rho, \boldsymbol{u}, T)=\frac{\rho}{(\pi T)^{3 / 2}} \exp \left(-\frac{\|\boldsymbol{\zeta}-\boldsymbol{u}\|^{2}}{T}\right), \\
& \rho=\int_{\mathbb{R}^{3}} f d \boldsymbol{\zeta}, \quad \boldsymbol{u}=\frac{1}{\rho} \int_{\mathbb{R}^{3}} \boldsymbol{\zeta} f d \boldsymbol{\zeta}, \quad T=\frac{2}{3 \rho} \int_{\mathbb{R}^{3}}\|\boldsymbol{\zeta}-\boldsymbol{u}\|^{2} f d \boldsymbol{\zeta},
\end{aligned}
$$

where each quantity is made dimensionless as follows using the reference time $t_{0}$, length $L$, density $\rho_{0}$, temperature $T_{0}$ and the most probable velocity of gas molecules, $c=\sqrt{2 R T_{0}}$ :

$$
t=\frac{\hat{t}}{t_{0}}, \quad \boldsymbol{x}=\frac{\hat{\boldsymbol{x}}}{L}, \quad \boldsymbol{\zeta}=\frac{\hat{\boldsymbol{\zeta}}}{c}, \quad f=\frac{\hat{f}}{\rho_{0} / c^{3}}, \quad f^{\mathrm{eq}}=\frac{\hat{f}^{\mathrm{eq}}}{\rho_{0} / c^{3}}, \quad \rho=\frac{\hat{\rho}}{\rho_{0}}, \quad \boldsymbol{u}=\frac{\hat{\boldsymbol{u}}}{c}, \quad T=\frac{\hat{T}}{T_{0}} .
$$

In Eq. (国), there are two dimensionless parameters: the Strouhal number, Sh, and the Knudsen number, Kn. The Strouhal number is the ratio of time scale between gas molecules and the system, that is, $\mathrm{Sh}=(L / c) / t_{0}$. The Knudsen number is the ratio of mean free path and system length, and defined as $\mathrm{Kn}=(2 / \sqrt{\pi}) c \tau_{0} / L$, where $\tau_{0}$ is the mean free time when $\hat{\rho}=\rho_{0}$. For the following optimization problem, $k$ is set as $(\sqrt{\pi} / 2) \mathrm{Kn}$.

\section{Optimization problem}

\subsection{Topology optimization}

The key idea of topology optimization is the replacement of a structural optimization problem with a material distribution problem, by introducing a fixed design domain, $D$, and a characteristic function, $\chi \in L^{\infty}(D ;\{0,1\})$. In flow channel design problems, the fluid (gas) domain $\Omega$ and the solid domain $D \backslash \Omega$ are usually expressed as shown in Fig. 四, where the characteristic function $\chi$ is defined as follows:

$$
\chi= \begin{cases}1 & \text { if } \boldsymbol{x} \in \Omega \\ 0 & \text { if } \boldsymbol{x} \in D \backslash \Omega\end{cases}
$$

The numerical treatment of this characteristic function is difficult because it can take 0 and 1 in infinitesimal regions repeatably. Various methods to overcome this difficulty have been proposed. Density methods, one type of these methods, are based on the convexification of the optimization problem. 


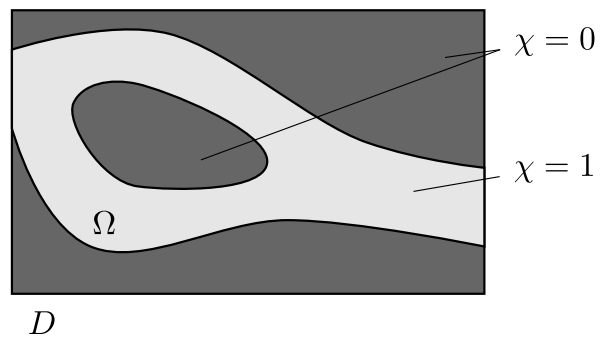

Figure 1: Conceptual schematic of topology optimization. A channel structure $\Omega$ is represented by the distribution of characteristic function $\chi$ in the design domain $D$, which contains the whole channel.

In density methods, the characteristic function $\chi \in L^{\infty}(D ;\{0,1\})$ is replaced with the normalized density, $\gamma \in L^{\infty}(D ;[0,1])$, which makes the design space of topology optimization convex. The convexification allows the existence of the grayscale regions, namely the intermediate state between the fluid and solid. Thus, a grayscale region usually implies that a porous medium can effectively work there, while it is often difficult for optimal structures including grayscale regions to be applied to actual product designs. To decrease grayscale area in the obtained optimal structures, various density-based topology optimization methods have been developed.

Among them, the density method based on a Heaviside projection with a PDE-based filter [52] is used in this work. According to this method, a scalar function, $\phi \in L^{\infty}(D ;[-1,1])$, is introduced as a design variable at first. Then, the design variable $\phi$ is regularized to decrease numerical instabilities, using the following Helmholtz PDE:

$$
-\omega^{2} \Delta \varphi+\varphi=\phi,
$$

where $\omega$ is a parameter adjusting the filter radius and $\varphi$ is the filtered variable. The normalized density $\gamma$ is finally defined as a smoothed Heaviside variable with the filtered variable $\varphi$ as follows:

$$
\gamma(\varphi)=\left\{\begin{array}{lll}
0 & \text { if } \quad-1 \leq \varphi<-w \\
\frac{1}{2}+\frac{15}{16}\left(\frac{\varphi}{w}\right)-\frac{5}{8}\left(\frac{\varphi}{w}\right)^{3}+\frac{15}{16}\left(\frac{\varphi}{w}\right)^{5} & \text { if }-w \leq \varphi \leq w \\
1 & \text { if } \quad w<\varphi \leq 1
\end{array}\right.
$$

where $w$ is the transition width from 0 to 1 .

\subsection{Extension of $B G K$ equation}

In topology optimization for NS flow problems, a fixed design domain is often considered to be composed of a porous medium so that fluid and solid domains can both be represented as the difference of their permeabilities, as proposed by Borravall and Petersson [24]. Based on Darcy's law, the fixed design domain is subjected to a fictitious force, $-\alpha(\gamma) \boldsymbol{u}$, where $\alpha(\gamma)$ is the inverse permeability. The fluid domains are represented as the domain with infinite permeability, so that $\alpha(1)=0$, while solid domains are represented as the domain with zero permeability, so that $\alpha(0) \rightarrow \infty$. When solving the NS equation with this force term included, a no-slip boundary condition is implicitly imposed on the fluid-solid interfaces. This method is efficient since it does not require cumbersome procedures of extracting the interfaces to impose boundary conditions at each optimization step.

However, the diffuse-reflection boundary condition to the velocity distribution function $f$, not the no-slip boundary condition to the flow velocity $\boldsymbol{u}$, is required on the gas-solid interfaces for the target problems in this work. To approximate the flow state with the diffuse-reflection boundary condition imposed on the interfaces, a similar formulation to those in several previous studies proposing topology optimization using LBM [43, 46], where the flow field is extended to solid domains, is constructed. Only the flow velocity in 
the solid domains is replaced with 0 based on the porosity model in those studies, while, in the proposed method, the temperature and Knudsen number in solid domains are additionally replaced as follows:

$$
\operatorname{Sh} \frac{\partial f}{\partial t}+\boldsymbol{\zeta} \cdot \nabla f=-\frac{\rho}{k_{\gamma}}\left(f-f^{\mathrm{eq}}\left(\rho, \boldsymbol{u}_{\gamma}, T_{\gamma}\right)\right)
$$

with

$$
\begin{aligned}
& \frac{1}{k_{\gamma}}=\gamma\left(\frac{1}{k}-\frac{1}{k_{s}}\right)+\frac{1}{k_{s}}, \\
& \boldsymbol{u}_{\gamma}(\boldsymbol{u}, \gamma)=\frac{\gamma(1+q)}{\gamma+q} \boldsymbol{u}, \\
& T_{\gamma}(T, \gamma)=\frac{\gamma(1+q)}{\gamma+q}\left(T-T_{s}\right)+T_{s},
\end{aligned}
$$

where $q$ is a parameter for controlling the curvature of $\boldsymbol{u}_{\gamma}$ and $T_{\gamma}$ with respect to $\gamma, k_{s}$ is a small value and $T_{s}$ is the temperature of the solid. The Knudsen number in the solid domains can be determined through some preliminary numerical confirmation before the optimization calculation. This formulation implies that solid is treated as an imaginary gas at temperature $T_{s}$ that immediately evolves to a static equilibrium state due to the small Knudsen number. Suitability of the above extension will be assessed numerically in Sec. 5.1 .

Multiplying Eq. (ए2) by $\left[1, \boldsymbol{\zeta},\|\boldsymbol{\zeta}\|^{2}\right]$, respectively, then integrating each of them over entire velocity space $\mathbb{R}^{3}$, the following three equations are obtained:

$$
\begin{aligned}
& \operatorname{Sh} \frac{\partial \rho}{\partial t}+\nabla \cdot(\rho \boldsymbol{u})=0, \\
& \operatorname{Sh} \frac{\partial(\rho \boldsymbol{u})}{\partial t}+\nabla \cdot\left(\frac{1}{2} \boldsymbol{p}+\rho \boldsymbol{u} \otimes \boldsymbol{u}\right)=\rho F, \\
& \operatorname{Sh} \frac{\partial}{\partial t}\left(\rho\left(\frac{3}{2} T+\|\boldsymbol{u}\|^{2}\right)\right)+\nabla \cdot\left(\rho \boldsymbol{u}\left(\frac{3}{2} T+\|\boldsymbol{u}\|^{2}\right)+\boldsymbol{p u}+\boldsymbol{q}\right)=\rho Q,
\end{aligned}
$$

with

$$
\begin{aligned}
& F=-\frac{\rho}{k_{\gamma}}\left(1-\frac{\gamma(1+q)}{\gamma+q}\right) \boldsymbol{u}, \\
& Q=-\frac{\rho}{k_{\gamma}}\left(\frac{3}{2}\left(1-\frac{\gamma(1+q)}{\gamma+q}\right)(T-T s)+\left(1-\left(\frac{\gamma(1+q)}{\gamma+q}\right)^{2}\right)\|\boldsymbol{u}\|^{2}\right),
\end{aligned}
$$

where $\boldsymbol{p}$ is the dimensionless stress tensor and $\boldsymbol{q}$ is the dimensionless heat-flow vector, defined by

$$
\boldsymbol{p}=2 \int_{\mathbb{R}^{3}}(\boldsymbol{\zeta}-\boldsymbol{u}) \otimes(\boldsymbol{\zeta}-\boldsymbol{u}) f d \boldsymbol{\zeta}, \quad \boldsymbol{q}=\int_{\mathbb{R}^{3}}(\boldsymbol{\zeta}-\boldsymbol{u})\|\boldsymbol{\zeta}-\boldsymbol{u}\|^{2} f d \boldsymbol{\zeta} .
$$

Eq. (펴) is the continuity equation, which implies that there is no mass flux on the gas-solid interfaces in the design domain. To explain this in detail, let us introduce a design domain $D$ including a gas-solid interface $\Gamma_{g s}$, as shown in Fig. 2 . The design domain $D$ can be divided into finite domains $\omega_{i}(i=1, \ldots, I)$ such that $D=\cup_{i=1}^{I} \omega_{i}$. The continuity equation holds everywhere in the design domain, so that the integration of the left-hand side of the continuity equation over the domain $\omega_{l}$ which includes a part of $\Gamma_{g s}$ also becomes 0 , as follows:

$$
\begin{aligned}
0=\int_{\omega_{l}} \nabla \cdot(\rho \boldsymbol{u}) d D & =\int_{\omega_{\text {gas }}} \nabla \cdot\left(\rho^{g} \boldsymbol{u}^{g}\right) d D+\int_{\omega_{\text {solid }}} \nabla \cdot\left(\rho^{s} \boldsymbol{u}^{s}\right) d D \\
& =-\int_{\partial \omega_{\text {gas }}} \rho^{g} \boldsymbol{u}^{g} \cdot \boldsymbol{n}^{g} d \Gamma-\int_{\partial \omega_{\text {solid }}} \rho^{s} \boldsymbol{u}^{s} \cdot \boldsymbol{n}^{s} d \Gamma \\
& =-\int_{\Gamma_{l}} \rho^{g} \boldsymbol{u}^{g} \cdot \boldsymbol{n}^{g} d \Gamma-\int_{\partial \omega_{\text {gas }} \backslash \Gamma_{l}} \rho^{g} \boldsymbol{u}^{g} \cdot \boldsymbol{n}^{g} d \Gamma-\int_{\partial \omega_{\text {solid }}} \rho^{s} \boldsymbol{u}^{s} \cdot \boldsymbol{n}^{s} d \Gamma,
\end{aligned}
$$




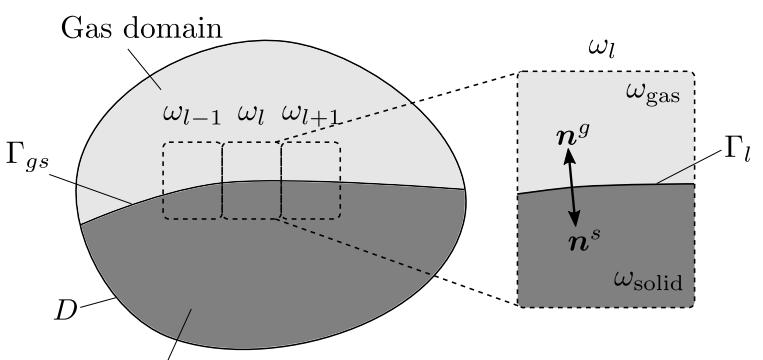

Solid domain

Figure 2: Schematic of a fixed design domain $D$ divided into finite domains $\omega_{i}(i=1, \ldots, I)$.

where $\omega_{l}=\omega_{\text {gas }} \cup \omega_{\text {solid }}, \omega_{\text {gas }}$ and $\omega_{\text {solid }}$ are the gas and solid domains, respectively, and $\Gamma_{l}=\Gamma_{g s} \cap \omega_{l}$. The quantities in $\omega_{\text {gas }}$ are denoted with the superscript $g$, and those in $\omega_{\text {solid }}$ are denoted with the superscript $s$. In the last line, the second term is canceled because the boundary integral on $\partial \omega_{\text {gas }} \backslash \Gamma_{l}$ with the normal vector pointing the opposite direction appears in the integration of the neighboring domains which share the boundary. The last term disappears since the flow velocity $\boldsymbol{u}_{s}$ approaches 0 . In the result, only the first term remains. Since $\omega_{l}$ can be sufficiently small, the following relation holds:

$$
\rho^{g} \boldsymbol{u}^{g} \cdot \boldsymbol{n}^{g}=0 \text { almost everywhere on } \Gamma_{g s},
$$

which is the zero mass flux condition that the flows on the gas-solid interfaces should satisfy. In addition, in the solid domain, the velocity distribution function $f(t, \boldsymbol{x}, \boldsymbol{\zeta})$ approaches the static equilibrium distribution function with temperature $T_{s}$, owing to the small Knudsen number. If $\left(\boldsymbol{\zeta} \cdot \boldsymbol{n}^{g}\right)>0$, the profile is advected to the gas domain, so that $\left.f(t, \boldsymbol{x}, \boldsymbol{\zeta})\right|_{\left(\boldsymbol{\zeta} \cdot \boldsymbol{n}^{g}\right)>0}$ at the nearest nodes to the interfaces in the gas domain results in having a similar profile to the static equilibrium distribution function.

On the other hand, Eqs. (파) and (펴) are equations for conservation of momentum and energy if $\gamma=1$. Otherwise, Eq. (파) implies that a fictitious force is imposed on solid domains in macroscopic view as existing methods for NS flow problems, and $\frac{\rho}{k_{\gamma}}\left(1-\frac{\gamma(1+q)}{\gamma+q}\right)$ is equivalent to $\alpha(\gamma)$ in those methods. Similarly, Eq. (며) implies that a fictitious heat generation or absorption occurs in solid domains.

The approximation of other boundary conditions, e.g. specular boundary condition and CercignaniLampis-Lord boundary condition, should also be considered, yet it is not discussed in this work and requires further research.

\subsection{Introduction of marginal distribution functions}

In this work, 2-D design problems for flow channels are considered. It implies that a cross-section of a channel with infinite depth along the $x_{3}$ axis is designed. Therefore it is assumed that all physical quantities are constant along the $x_{3}$ axis and the flow velocity $u_{3}$ maintains a null value. Moreover, based on Chu's method [52], $\zeta_{3}$ can be removed from the BGK equation (ए2) by introducing the following marginal distribution functions, $f_{1}$ and $f_{2}$ :

$$
\begin{aligned}
& f_{1}\left(t, x_{1}, x_{2}, \zeta_{1}, \zeta_{2}\right)=\int_{-\infty}^{\infty} f\left(t, x_{1}, x_{2}, \zeta_{1}, \zeta_{2}, \zeta_{3}\right) d \zeta_{3} \\
& f_{2}\left(t, x_{1}, x_{2}, \zeta_{1}, \zeta_{2}\right)=\int_{-\infty}^{\infty} \zeta_{3}^{2} f\left(t, x_{1}, x_{2}, \zeta_{1}, \zeta_{2}, \zeta_{3}\right) d \zeta_{3} .
\end{aligned}
$$

Specifically, multiplying the BGK equation by 1 and $\zeta_{3}^{2}$ and integrating the results over the space of $\zeta_{3}$ $(-\infty, \infty)$, respectively, two similar equations, namely the governing equations of the marginal distribution 
functions, are obtained as follows:

$$
\begin{aligned}
& \operatorname{Sh} \frac{\partial \boldsymbol{f}}{\partial t}+(\boldsymbol{\zeta} \cdot \nabla) \boldsymbol{f}=-\frac{\rho}{k_{\gamma}}\left(\boldsymbol{f}-\boldsymbol{f}^{\mathrm{eq}}\left(\rho, \boldsymbol{u}_{\gamma}, T_{\gamma}\right)\right) \\
& \boldsymbol{f}^{\mathrm{eq}}\left(\rho, \boldsymbol{u}_{\gamma}, T_{\gamma}\right)=\left[\begin{array}{c}
1 \\
T_{\gamma} / 2
\end{array}\right] \frac{\rho}{\pi T_{\gamma}} \exp \left(-\frac{\left\|\boldsymbol{\zeta}-\boldsymbol{u}_{\gamma}\right\|^{2}}{T_{\gamma}}\right), \\
& \rho=\int_{\mathbb{R}^{2}} \boldsymbol{a}_{1} \cdot \boldsymbol{f} d \boldsymbol{\zeta}, \quad \boldsymbol{u}=\frac{1}{\rho} \int_{\mathbb{R}^{2}} \boldsymbol{\zeta}\left(\boldsymbol{a}_{1} \cdot \boldsymbol{f}\right) d \boldsymbol{\zeta}, \quad T=\frac{2}{3 \rho} \int_{\mathbb{R}^{2}}\left(\|\boldsymbol{\zeta}-\boldsymbol{u}\|^{2} \boldsymbol{a}_{1}+\boldsymbol{a}_{2}\right) \cdot \boldsymbol{f} d \boldsymbol{\zeta},
\end{aligned}
$$

with

$$
\boldsymbol{f}=\left[\begin{array}{l}
f_{1} \\
f_{2}
\end{array}\right], \boldsymbol{a}_{1}=\left[\begin{array}{l}
1 \\
0
\end{array}\right], \boldsymbol{a}_{2}=\left[\begin{array}{l}
0 \\
1
\end{array}\right] .
$$

It is noted that all physical vector quantities, such as $\boldsymbol{x}, \boldsymbol{\zeta}$ and $\boldsymbol{u}$, belong to $\mathbb{R}^{2}$.

\subsection{Optimization problem formulation}

An optimization problem for a flow channel design with a volume constraint is formulated. The performance of the flow channel is evaluated in the evaluation domain $\Omega_{e}$, on the inlet $\Gamma_{\text {in }}$ and on the outlet $\Gamma_{\text {out }}$ as shown in Fig. $\mathbf{3}$, during the period $\Theta=\left[t_{i}, t_{f}\right]$, using macroscopic quantities $\rho, \boldsymbol{u}$ and $T$. The evaluation domain is defined as the non-design domain where the design variable does not vary. Therefore,

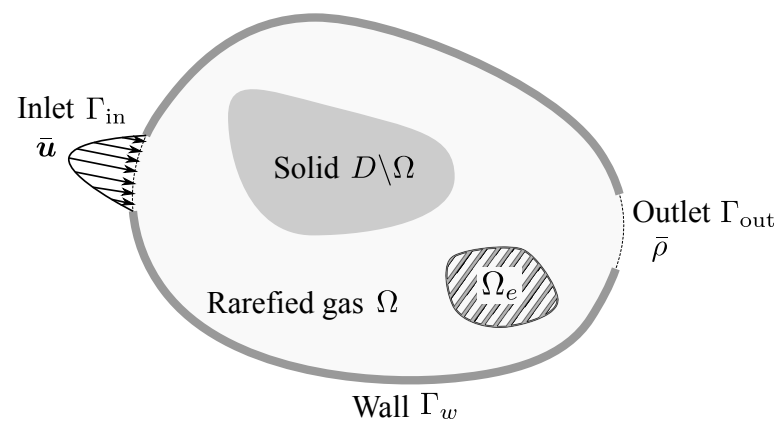

Figure 3: Domain settings and boundary conditions.

the optimization problem is described as

$$
\begin{aligned}
\inf _{\phi} \quad J & =\int_{\Theta} \int_{\Omega_{e}} j_{e}(\rho, \boldsymbol{u}, T) d D d t+\int_{\Theta} \int_{\Gamma_{\mathrm{in}}} j_{\text {in }}(\rho, \boldsymbol{u}, T) d \Gamma d t+\int_{\Theta} \int_{\Gamma_{\text {out }}} j_{\text {out }}(\rho, \boldsymbol{u}, T) d \Gamma d t, \\
\text { subject to } & V-V_{\max } \leq 0
\end{aligned}
$$

where $J$ is the objective functional, $V$ is the area of rarefied gas domains and $V_{\max }$ is the upper limit of the area. To make the description general, the functions $j_{e}, j_{\text {in }}$ and $j_{\text {out }}$ are used as the integrands of the objective functional. The rarefied gas flow is described by the following initial-boundary value problem:

$$
\begin{array}{ll}
\text { Sh } \frac{\partial \boldsymbol{f}}{\partial t}+(\boldsymbol{\zeta} \cdot \nabla) \boldsymbol{f}=-\frac{\rho}{k_{\gamma}}\left(\boldsymbol{f}-\boldsymbol{f}^{\mathrm{eq}}\left(\rho, \boldsymbol{u}_{\gamma}, T_{\gamma}\right)\right) & \text { in } \Theta \times D \times \mathbb{R}^{2}, \\
\boldsymbol{f}\left(t_{i}, \boldsymbol{x}, \boldsymbol{\zeta}\right)=\boldsymbol{f}_{0} & \text { in } D \times \mathbb{R}^{2}, \\
\boldsymbol{f}=\boldsymbol{f}^{\mathrm{eq}}\left(\sigma_{w}, \mathbf{0}, T_{w}\right) & \text { in } \Theta \times \Gamma_{w} \times \mathbb{R}_{+}^{2}, \\
\boldsymbol{f}=\boldsymbol{f}^{\mathrm{eq}}\left(\sigma_{\text {in }}, \mathbf{0}, T_{\text {in }}\right) & \text { in } \Theta \times \Gamma_{\text {in }} \times \mathbb{R}_{+}^{2}, \\
\boldsymbol{f}=\boldsymbol{f}^{\mathrm{eq}}\left(\sigma_{\text {out }}, \mathbf{0}, T_{\text {out }}\right) & \text { in } \Theta \times \Gamma_{\text {out }} \times \mathbb{R}_{+}^{2},
\end{array}
$$


where

$$
\begin{aligned}
& \sigma_{w}=-2 \sqrt{\frac{\pi}{T_{w}}} \int_{\mathbb{R}_{-}^{2}}(\boldsymbol{\zeta} \cdot \boldsymbol{n})\left(\boldsymbol{a}_{1} \cdot \boldsymbol{f}\right) d \boldsymbol{\zeta}, \\
& \sigma_{\mathrm{in}}=\frac{2}{\overline{\boldsymbol{u}} \cdot \boldsymbol{n}-\sqrt{T_{\mathrm{in}} / \pi}} \int_{\mathbb{R}_{-}^{2}}(\boldsymbol{\zeta}-\overline{\boldsymbol{u}}) \cdot \boldsymbol{n}\left(\boldsymbol{a}_{1} \cdot \boldsymbol{f}\right) d \boldsymbol{\zeta}, \\
& \sigma_{\text {out }}=2\left(\bar{\rho}-\int_{\mathbb{R}_{-}^{2}} \boldsymbol{a}_{1} \cdot \boldsymbol{f} d \boldsymbol{\zeta}\right) .
\end{aligned}
$$

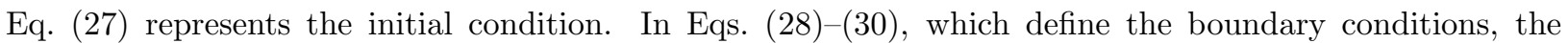
velocity space $\mathbb{R}_{+}^{2}=\left\{\boldsymbol{\zeta} \in \mathbb{R}^{2} \mid \boldsymbol{\zeta} \cdot \boldsymbol{n} \gtrless 0\right\}$ and $\boldsymbol{n}$ indicates the normal unit vector directed inward on the

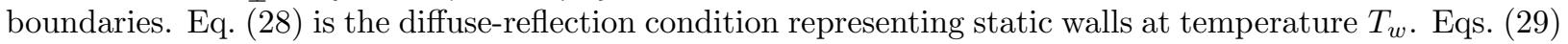
and (Bत) express the inlet boundary condition prescribing the flow velocity as $\overline{\boldsymbol{u}}$ and the outlet boundary condition prescribing the density as $\bar{\rho}$, where the gas outside the fixed design domain is assumed to be in a static equilibrium state at temperature $T_{\text {in }}$ and $T_{\text {out }}$, respectively.

\section{Numerical implementation}

Here, the optimization algorithm is firstly explained. Then, the outline of the sensitivity analysis, which is written in detail in Appendix A, is described. Finally, the details of the flow field analysis in this study are explained.

\subsection{Optimization algorithm}

The optimization algorithm of the proposed method is as below:

Step 1. The design variable $\phi$ is initialized.

Step 2. The normalized density $\gamma$ is calculated through Eqs. (四) and (四).

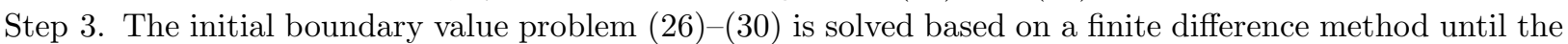
steady state is achieved. The details of this analysis are described in Sec. 4.3.3.

Step 4. If the objective functional $J$ is converged, the optimization is finished. Otherwise, the adjoint initial boundary value problem described later is solved.

Step 5. The design sensitivity is calculated.

Step 6. The design variable is updated using the method of moving asymptotes (MMA) [5.3]. After that, the procedure is returned to Step 2.

The convergence of the objective functional is judged by satisfying the following condition twice in a row:

$$
\frac{\left|J^{\left(n_{\mathrm{opt}}\right)}-J^{\left(n_{\mathrm{opt}}-1\right)}\right|}{\left|J^{\left(n_{\mathrm{opt}}\right)}\right|}<\varepsilon_{\mathrm{opt}},
$$

where the superscript $n_{\mathrm{opt}}$ is the iteration number of the optimization loop and $\varepsilon_{\mathrm{opt}}$ represents the judgment criterion.

\subsection{Sensitivity analysis}

In this section, the sensitivity analysis based on the Lagrange multiplier method and the adjoint variable method is described. At first, the governing equation, initial condition and boundary conditions are considered as the equality constraints of the optimization problem, and the problem is replaced with an unconstrained problem using the Lagrange multiplier method as follows:

$$
\inf _{\gamma} \mathcal{L}=J+G+I+B_{w}+B_{\text {in }}+B_{\text {out }}+M_{D}+M_{B}+\mu\left(V-V_{\text {max }}\right),
$$


where

$$
\begin{aligned}
& J=\int_{\Theta} \int_{\Omega_{e}} j_{e}(\tilde{\rho}, \tilde{\boldsymbol{u}}, \tilde{T}) d D d t+\int_{\Theta} \int_{\Gamma_{\text {in }}} j_{\text {in }}(\tilde{\rho}, \tilde{\boldsymbol{u}}, \tilde{T}) d \Gamma d t+\int_{\Theta} \int_{\Gamma_{\text {out }}} j_{\text {out }}(\tilde{\rho}, \tilde{\boldsymbol{u}}, \tilde{T}) d \Gamma d t \\
& G=\int_{\Theta} \int_{D} \int_{\mathbb{R}^{2}} \tilde{\boldsymbol{g}} \cdot\left(\operatorname{Sh} \frac{\partial \tilde{\boldsymbol{f}}}{\partial t}+(\boldsymbol{\zeta} \cdot \nabla) \tilde{\boldsymbol{f}}+\frac{\tilde{\rho}}{k_{\gamma}}\left(\tilde{\boldsymbol{f}}-\boldsymbol{f}^{\mathrm{eq}}\left(\tilde{\rho}, \tilde{\boldsymbol{u}}_{\gamma}, \tilde{T}_{\gamma}\right)\right)\right) d \boldsymbol{\zeta} d D d t, \\
& I=\int_{D} \int_{\mathbb{R}^{2}} \tilde{\boldsymbol{g}}_{0} \cdot\left(\tilde{\boldsymbol{f}}\left(t_{i}, \boldsymbol{x}, \boldsymbol{\zeta}\right)-\boldsymbol{f}_{0}\right) d \boldsymbol{\zeta} d D, \\
& B_{w}=\int_{\Theta} \int_{\Gamma_{w}} \int_{\mathbb{R}_{+}^{2}} \tilde{\boldsymbol{g}}_{w} \cdot\left(\tilde{\boldsymbol{f}}-\boldsymbol{f}^{\mathrm{eq}}\left(\tilde{\sigma}_{w}, \mathbf{0}, T_{w}\right)\right) d \boldsymbol{\zeta} d \Gamma d t \\
& +\int_{\Theta} \int_{\Gamma_{w}} \tilde{s}_{w}\left(\tilde{\sigma}_{w}+2 \sqrt{\frac{\pi}{T_{w}}} \int_{\mathbb{R}_{-}^{2}}(\boldsymbol{\zeta} \cdot \boldsymbol{n})\left(\boldsymbol{a}_{1} \cdot \tilde{\boldsymbol{f}}\right) d \boldsymbol{\zeta}\right) d \Gamma d t, \\
& B_{\text {in }}=\int_{\Theta} \int_{\Gamma_{\text {in }}} \int_{\mathbb{R}_{+}^{2}} \tilde{\boldsymbol{g}}_{\text {in }} \cdot\left(\tilde{\boldsymbol{f}}-\boldsymbol{f}^{\mathrm{eq}}\left(\tilde{\sigma}_{\mathrm{in}}, \mathbf{0}, T_{\mathrm{in}}\right)\right) d \boldsymbol{\zeta} d \Gamma d t \\
& +\int_{\Theta} \int_{\Gamma_{\mathrm{in}}} \tilde{s}_{\mathrm{in}}\left(\tilde{\sigma}_{\mathrm{in}}-\frac{2}{\overline{\boldsymbol{u}} \cdot \boldsymbol{n}-\sqrt{\frac{T_{\mathrm{in}}}{\pi}}} \int_{\mathbb{R}_{-}^{2}}(\boldsymbol{\zeta}-\overline{\boldsymbol{u}}) \cdot \boldsymbol{n}\left(\boldsymbol{a}_{1} \cdot \tilde{\boldsymbol{f}}\right) d \boldsymbol{\zeta}\right) d \Gamma d t, \\
& B_{\text {out }}=\int_{\Theta} \int_{\Gamma_{\text {out }}} \int_{\mathbb{R}_{+}^{2}} \tilde{\boldsymbol{g}}_{\text {out }} \cdot\left(\tilde{\boldsymbol{f}}-\boldsymbol{f}^{\text {eq }}\left(\tilde{\sigma}_{\text {out }}, \mathbf{0}, T_{\text {out }}\right)\right) d \boldsymbol{\zeta} d \Gamma d t \\
& +\int_{\Theta} \int_{\Gamma_{\text {out }}} \tilde{s}_{\text {out }}\left(\tilde{\sigma}_{\text {out }}-2\left(\bar{\rho}-\int_{\mathbb{R}_{-}^{2}} \boldsymbol{a}_{1} \cdot \tilde{\boldsymbol{f}} d \boldsymbol{\zeta}\right)\right) d \Gamma d t, \\
& M_{D}=\int_{\Theta} \int_{D} \tilde{r}_{D}\left(\tilde{\rho}-\int_{\mathbb{R}^{2}} \boldsymbol{a}_{1} \cdot \tilde{\boldsymbol{f}} d \boldsymbol{\zeta}\right) d D d t+\int_{\Theta} \int_{D} \tilde{\boldsymbol{v}}_{D} \cdot\left(\tilde{\boldsymbol{u}}-\frac{1}{\tilde{\rho}} \int_{\mathbb{R}^{2}} \boldsymbol{\zeta}\left(\boldsymbol{a}_{1} \cdot \tilde{\boldsymbol{f}}\right) d \boldsymbol{\zeta}\right) d D d t \\
& +\int_{\Theta} \int_{D} \tilde{\theta}_{D}\left(\tilde{T}-\frac{2}{3 \tilde{\rho}} \int_{\mathbb{R}^{2}}\left(\|\boldsymbol{\zeta}-\tilde{\boldsymbol{u}}\|^{2} \boldsymbol{a}_{1}+\boldsymbol{a}_{2}\right) \cdot \tilde{\boldsymbol{f}} d \boldsymbol{\zeta}\right) d D d t, \\
& M_{B}=\int_{\Theta} \int_{\partial D} \tilde{r}_{B}\left(\tilde{\rho}-\int_{\mathbb{R}^{2}} \boldsymbol{a}_{1} \cdot \tilde{\boldsymbol{f}} d \boldsymbol{\zeta}\right) d D d t+\int_{\Theta} \int_{\partial D} \tilde{\boldsymbol{v}}_{B} \cdot\left(\tilde{\boldsymbol{u}}-\frac{1}{\tilde{\rho}} \int_{\mathbb{R}^{2}} \boldsymbol{\zeta}\left(\boldsymbol{a}_{1} \cdot \tilde{\boldsymbol{f}}\right) d \boldsymbol{\zeta}\right) d D d t \\
& +\int_{\Theta} \int_{\partial D} \tilde{\theta}_{B}\left(\tilde{T}-\frac{2}{3 \tilde{\rho}} \int_{\mathbb{R}^{2}}\left(\|\boldsymbol{\zeta}-\tilde{\boldsymbol{u}}\|^{2} \boldsymbol{a}_{1}+\boldsymbol{a}_{2}\right) \cdot \tilde{\boldsymbol{f}} d \boldsymbol{\zeta}\right) d D d t \\
& V=\int_{D} \gamma d D
\end{aligned}
$$

where $\tilde{\Psi}_{\text {st }}=\left\{\tilde{\boldsymbol{f}}, \tilde{\rho}, \tilde{\boldsymbol{u}}, \tilde{T}, \tilde{\sigma}_{w}, \tilde{\sigma}_{\text {in }}, \tilde{\sigma}_{\text {out }}\right\}$ are variables used in the Lagrangian and $\tilde{\Psi}_{\text {ad }}=\left\{\tilde{\boldsymbol{g}}, \tilde{\boldsymbol{g}}_{0}, \tilde{\boldsymbol{g}}_{w}, \tilde{s}_{w}, \tilde{\boldsymbol{g}}_{\text {in }}, \tilde{s}_{\text {in }}, \tilde{\boldsymbol{g}}_{\text {out }}, \tilde{s}_{\text {out }}, \tilde{r}_{D}, \tilde{\boldsymbol{v}}\right.$ are the Lagrange multipliers. Besides, the notations $\tilde{\boldsymbol{u}}_{\gamma}=\boldsymbol{u}_{\gamma}(\tilde{\boldsymbol{u}}, \gamma)$ and $\tilde{T}_{\gamma}=T_{\gamma}(\tilde{T}, \gamma)$ are introduced for the simple description.

The Lagrangian should satisfy the following necessary conditions at the optimal result:

$$
\begin{array}{ll}
d \mathcal{L}\left(\tilde{\psi}_{\mathrm{ad}} ; \delta \tilde{\psi}_{\mathrm{ad}}\right)=0 & \text { for } \tilde{\psi}_{\mathrm{ad}} \in \tilde{\Psi}_{\mathrm{ad}}, \\
d \mathcal{L}\left(\tilde{\psi}_{\mathrm{st}} ; \delta \tilde{\psi}_{\mathrm{st}}\right)=0 & \text { for } \tilde{\psi}_{\mathrm{st}} \in \tilde{\Psi}_{\mathrm{st}} .
\end{array}
$$

The optimal conditions for Lagrange multipliers (44) coincide with the initial-boundary value problem ([26)(301). On the other hand, the optimal conditions for the variables (45) result in the following adjoint 
initial-boundary value problem:

$$
\begin{aligned}
& -\operatorname{Sh} \frac{\partial \boldsymbol{g}}{\partial t}-(\boldsymbol{\zeta} \cdot \nabla) \boldsymbol{g}=-\frac{\rho}{k_{\gamma}}\left(\boldsymbol{g}-\boldsymbol{g}^{\mathrm{eq}}\left(\rho_{\mathrm{ad}}, \boldsymbol{u}_{\mathrm{ad}}, T_{\mathrm{ad}}\right)\right) \quad \text { in } \Theta \times D \times \mathbb{R}^{2}, \\
& \boldsymbol{g}\left(t_{f}, \boldsymbol{x}, \boldsymbol{\zeta}\right)=0 \quad \text { in } D \times \mathbb{R}^{2}, \\
& \boldsymbol{g}=2 s_{w} \sqrt{\frac{\pi}{T_{w}}} \boldsymbol{a}_{1} \\
& \text { in } \Theta \times \Gamma_{w} \times \mathbb{R}_{-}^{2}, \\
& (\boldsymbol{\zeta} \cdot \boldsymbol{n}) \boldsymbol{g}=-\frac{2 s_{\mathrm{in}}(\boldsymbol{\zeta}-\overline{\boldsymbol{u}}) \cdot \boldsymbol{n}}{\overline{\boldsymbol{u}} \cdot \boldsymbol{n}-\sqrt{T_{\mathrm{in}} / \pi}} \boldsymbol{a}_{1}-\boldsymbol{g}^{\mathrm{eq}}\left(r_{B}, \boldsymbol{v}_{B}, \theta_{B}\right) \quad \text { in } \Theta \times \Gamma_{\mathrm{in}} \times \mathbb{R}_{-}^{2}, \\
& (\boldsymbol{\zeta} \cdot \boldsymbol{n}) \boldsymbol{g}=2 s_{\text {out }} \boldsymbol{a}_{1}-\boldsymbol{g}^{\mathrm{eq}}\left(r_{B}, \boldsymbol{v}_{B}, \theta_{B}\right) \quad \text { in } \Theta \times \Gamma_{\text {out }} \times \mathbb{R}_{-}^{2},
\end{aligned}
$$

where

$$
\begin{aligned}
& \boldsymbol{g}^{\mathrm{eq}}(r, \boldsymbol{v}, \theta)=r \boldsymbol{a}_{1}+\frac{1}{\rho}(\boldsymbol{\zeta} \cdot \boldsymbol{v}) \boldsymbol{a}_{1}+\frac{2 \theta}{3 \rho}\left(\|\boldsymbol{\zeta}-\boldsymbol{u}\|^{2} \boldsymbol{a}_{1}+\boldsymbol{a}_{2}\right), \\
& \rho_{\mathrm{ad}}=\frac{k_{\gamma}}{\rho} r_{D}=\left\{\begin{array}{ll}
-\frac{1}{\rho} \int_{\mathbb{R}^{2}} \boldsymbol{g} \cdot\left(\boldsymbol{f}-2 \boldsymbol{f}_{\gamma}^{\mathrm{eq}}\right) d \boldsymbol{\zeta}-\frac{1}{\rho}\left(\boldsymbol{u}_{\mathrm{ad}} \cdot \boldsymbol{u}\right)-\frac{1}{\rho} T_{\mathrm{ad}} T & \text { in } \Theta \times D \backslash \Omega_{e}, \\
-\frac{1}{\rho} \int_{\mathbb{R}^{2}} \boldsymbol{g} \cdot\left(\boldsymbol{f}-2 \boldsymbol{f}_{\gamma}^{\text {eq }}\right) d \boldsymbol{\zeta}-\frac{1}{\rho}\left(\boldsymbol{u}_{\mathrm{ad}} \cdot \boldsymbol{u}\right)-\frac{1}{\rho} T_{\mathrm{ad}} T-\frac{k_{\gamma}}{\rho} \frac{\partial j_{e}}{\partial \rho} & \text { in } \quad \Theta \times \Omega_{e}
\end{array},\right. \\
& \boldsymbol{u}_{\mathrm{ad}}=\frac{k_{\gamma}}{\rho} \boldsymbol{v}_{D}= \begin{cases}\frac{2}{T_{\gamma}} \frac{\gamma(1+q)}{\gamma+q} \int_{\mathbb{R}^{2}}\left(\boldsymbol{g} \cdot \boldsymbol{f}_{\gamma}^{\mathrm{eq}}\right)(\boldsymbol{\zeta}-\boldsymbol{u}) d \boldsymbol{\zeta} & \text { in } \Theta \times D \backslash \Omega_{e}, \\
\frac{2}{T_{\gamma}} \frac{\gamma(1+q)}{\gamma+q} \int_{\mathbb{R}^{2}}\left(\boldsymbol{g} \cdot \boldsymbol{f}_{\gamma}^{\mathrm{eq}}\right)(\boldsymbol{\zeta}-\boldsymbol{u}) d \boldsymbol{\zeta}-\frac{k_{\gamma}}{\rho} \frac{\partial j_{e}}{\partial \boldsymbol{u}} & \text { in } \Theta \times \Omega_{e},\end{cases}
\end{aligned}
$$

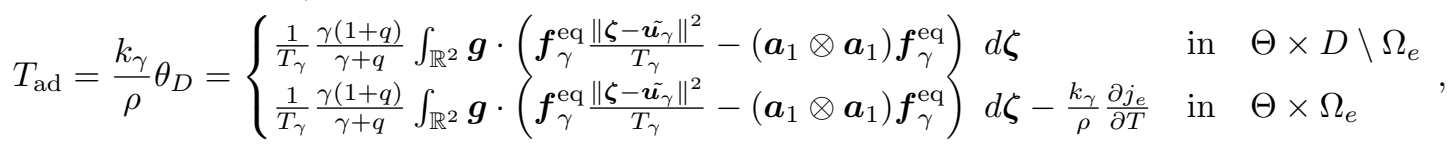

$$
\begin{aligned}
& r_{B}=\left\{\begin{array}{ll}
-\frac{\partial j_{\text {in }}}{\partial \rho}-\frac{1}{\rho}\left(\boldsymbol{v}_{B} \cdot \boldsymbol{u}\right)-\frac{1}{\rho} \theta_{B} T & \text { in } \Theta \times \Gamma_{\text {in }} \\
-\frac{\partial j_{\text {out }}}{\partial \rho}-\frac{1}{\rho}\left(\boldsymbol{v}_{B} \cdot \boldsymbol{u}\right)-\frac{1}{\rho} \theta_{B} T & \text { in } \Theta \times \Gamma_{\text {out }}
\end{array},\right. \\
& \boldsymbol{v}_{B}=\left\{\begin{array}{ll}
-\frac{\partial j_{\text {in }}}{\partial u} & \text { in } \Theta \times \Gamma_{\text {in }} \\
-\frac{\partial j_{\text {out }}}{\partial \boldsymbol{u}} & \text { in } \Theta \times \Gamma_{\text {out }}
\end{array},\right. \\
& \theta_{B}=\left\{\begin{array}{ll}
-\frac{\partial j_{\text {in }}}{\partial T} & \text { in } \Theta \times \Gamma_{\text {in }} \\
-\frac{\partial j_{\text {out }}}{\partial T} & \text { in } \Theta \times \Gamma_{\text {out }}
\end{array},\right. \\
& s_{w}=\frac{1}{\sigma_{w}} \int_{\mathbb{R}_{+}^{2}}(\boldsymbol{\zeta} \cdot \boldsymbol{n})\left(\boldsymbol{g} \cdot \boldsymbol{f}^{\mathrm{eq}}\left(\sigma_{w}, \mathbf{0}, T_{w}\right)\right) d \boldsymbol{\zeta} \quad \text { in } \Theta \times \Gamma_{w}, \\
& s_{\text {in }}=\frac{1}{\sigma_{\text {in }}} \int_{\mathbb{R}_{+}^{2}}\left((\boldsymbol{\zeta} \cdot \boldsymbol{n}) \boldsymbol{g}+\boldsymbol{g}^{\mathrm{eq}}\left(r_{B}, \boldsymbol{v}_{B}, \theta_{B}\right)\right) \cdot \boldsymbol{f}^{\mathrm{eq}}\left(\sigma_{\mathrm{in}}, \mathbf{0}, T_{\mathrm{in}}\right) d \boldsymbol{\zeta} \quad \text { in } \Theta \times \Gamma_{\mathrm{in}}, \\
& s_{\text {out }}=\frac{1}{\sigma_{\text {out }}} \int_{\mathbb{R}_{+}^{2}}\left((\boldsymbol{\zeta} \cdot \boldsymbol{n}) \boldsymbol{g}+\boldsymbol{g}^{\mathrm{eq}}\left(r_{B}, \boldsymbol{v}_{B}, \theta_{B}\right)\right) \cdot \boldsymbol{f}^{\mathrm{eq}}\left(\sigma_{\text {out }}, \mathbf{0}, T_{\text {out }}\right) d \boldsymbol{\zeta} \quad \text { in } \Theta \times \Gamma_{\text {out }},
\end{aligned}
$$

with the notation $\boldsymbol{f}_{\gamma}^{\mathrm{eq}}=\boldsymbol{f}^{\mathrm{eq}}\left(\rho, \boldsymbol{u}_{\gamma}, T_{\gamma}\right)$. In this adjoint field, the time retrogrades from the final time $t_{f}$ to the initial time $t_{i}$. The details for the derivation of the adjoint equation are described in Appendix A.

Substituting these optimal points, that is, substituting $\tilde{\psi}_{\text {st }}=\psi_{\text {st }}$ for all $\tilde{\psi}_{\text {st }} \in \tilde{\Psi}_{\text {st }}$ and $\tilde{\psi}_{\text {ad }}=\psi_{\text {ad }}$ for all $\tilde{\psi}_{\text {ad }} \in \tilde{\Psi}_{\text {ad }}$ in the Lagrangian, the gradient with respect to the normalized density, $\mathcal{L}_{\gamma}^{\prime}$, is obtained as 
Gâteaux derivative of the Lagrangian at $\gamma$ as below:

$$
\begin{aligned}
\left\langle\mathcal{L}_{\gamma}^{\prime}, \delta \gamma\right\rangle & \equiv d \mathcal{L}(\gamma ; \delta \gamma) \\
& =\int_{D}\left(\int _ { \Theta } \int _ { \mathbb { R } ^ { 2 } } \left(\rho\left(\frac{1}{k}-\frac{1}{k_{s}}\right) \boldsymbol{g} \cdot\left(\boldsymbol{f}-\boldsymbol{f}_{\gamma}^{\mathrm{eq}}\right)\right.\right. \\
& -\frac{q(1+q)}{(\gamma+q)^{2}} \frac{\rho}{k_{\gamma}} \frac{2\left(\boldsymbol{\zeta}-\boldsymbol{u}_{\gamma}\right) \cdot \boldsymbol{u}}{T_{\gamma}} \boldsymbol{g} \cdot \boldsymbol{f}_{\gamma}^{\mathrm{eq}} \\
& \left.\left.-\frac{q(1+q)}{(\gamma+q)^{2}} \frac{\rho}{k_{\gamma}} \frac{T-T_{s}}{T_{\gamma}} \boldsymbol{g} \cdot\left(\frac{\left\|\boldsymbol{\zeta}-\boldsymbol{u}_{\gamma}\right\|^{2}}{T_{\gamma}} \boldsymbol{f}_{\gamma}^{\mathrm{eq}}-\left(\boldsymbol{a}_{1} \otimes \boldsymbol{a}_{1}\right) \boldsymbol{f}_{\gamma}^{\mathrm{eq}}\right)\right) d \boldsymbol{\zeta} d t+\mu\right) \delta \gamma d D .
\end{aligned}
$$

In time-dependent problems, the values of both state and adjoint variables should generally be stored at every time to calculate this gradient, which causes high computational costs. If a sufficiently long period from initial time $t_{i}$ to final time $t_{f}$ is set, the values of state and adjoint variables stay unchanged at almost all time and the integrand of gradient can be considered as constant with regard to time. Therefore, the gradient can be approximated by using the values of both state and adjoint variables at their steady states as below:

$$
\begin{gathered}
\mathcal{L}_{\gamma}^{\prime} \approx\left(t_{f}-t_{i}\right) \int_{\mathbb{R}^{2}}\left(\rho\left(\frac{1}{k}-\frac{1}{k_{s}}\right) \boldsymbol{g} \cdot\left(\boldsymbol{f}-\boldsymbol{f}_{\gamma}^{\mathrm{eq}}\right)-\frac{q(1+q)}{(\gamma+q)^{2}} \frac{\rho}{k_{\gamma}} \frac{2\left(\boldsymbol{\zeta}-\boldsymbol{u}_{\gamma}\right) \cdot \boldsymbol{u}}{T_{\gamma}} \boldsymbol{g} \cdot \boldsymbol{f}_{\gamma}^{\mathrm{eq}}\right. \\
\left.-\frac{q(1+q)}{(\gamma+q)^{2}} \frac{\rho}{k_{\gamma}} \frac{T-T_{s}}{T_{\gamma}} \boldsymbol{g} \cdot\left(\frac{\left\|\boldsymbol{\zeta}-\boldsymbol{u}_{\gamma}\right\|^{2}}{T_{\gamma}} \boldsymbol{f}_{\gamma}^{\mathrm{eq}}-\left(\boldsymbol{a}_{1} \otimes \boldsymbol{a}_{1}\right) \boldsymbol{f}_{\gamma}^{\mathrm{eq}}\right)\right) d \boldsymbol{\zeta}+\mu
\end{gathered}
$$

In this study, the above approximated gradient (52) is adopted in the optimization process instead of the original gradient (5]). This also means that the objective functional is evaluated only in the steady state. According to the Heaviside projection method [52], the design sensitivity, $\mathcal{L}_{\phi}^{\prime}$, which is the gradient with respect to the design variable $\phi$, can be obtained by filtering the gradient $\mathcal{L}_{\gamma}^{\prime}$ as follows:

$$
-\omega^{2} \Delta \mathcal{L}_{\phi}^{\prime}+\mathcal{L}_{\phi}^{\prime}=\frac{d \gamma}{d \varphi} \mathcal{L}_{\gamma}^{\prime}
$$

\subsection{Details of flow field analysis}

In the analysis of state and adjoint flow fields, the fixed design domain $D$ is discretized on a grid. The space step, $\Delta x$, is common in both $x_{1}$ and $x_{2}$ directions and is determined so that it is smaller than the mean free path of gas. The velocity space is also discretized on a grid after the space which is originally infinite is replaced to the finite space $D_{\zeta}=\left\{\boldsymbol{\zeta} \in \mathbb{R}^{2} \mid-Z \leq \zeta_{1}, \zeta_{2} \leq Z\right\}$, where $Z$ is the threshold. Based on the time splitting method [54], the computation of the governing and adjoint equation is divided into two steps: the advection step and collision step. At first, the Lax-Wendroff scheme, an explicit finite difference scheme, with a flux-limiter function is used to compute the advection step. Due to the explicit scheme, the time step $\Delta t$ has to be determined so that it satisfies the CFL condition, and it is defined by

$$
\Delta t=\frac{1}{2} \frac{\operatorname{Sh} \Delta x}{2 Z}
$$

which is a quarter of the time step allowed by CFL condition. Then, the collision step is computed by solving the following ordinary differential equation with regard to time on the grid of real and velocity spaces:

$$
\begin{aligned}
\frac{d f(t)}{d t} & =-\frac{\rho^{*}}{k}\left(f(t)-f^{\mathrm{eq}}\left(\rho^{*}, \boldsymbol{u}^{*}, T^{*}\right)\right), \\
f(0) & =f^{*},
\end{aligned}
$$

where the superscript ${ }^{*}$ denotes the post-advection value. 
For convergence judgment in the state flow field, moments of velocity distribution function, namely, density $\rho$, momentum $\boldsymbol{M}$ and total energy $E$ are used. The momentum and total energy are defined by

$$
\begin{aligned}
\boldsymbol{M} & =\int_{\mathbb{R}^{2}} \boldsymbol{\zeta}\left(\boldsymbol{a}_{1} \cdot \boldsymbol{f}\right) d \boldsymbol{\zeta}=\rho \boldsymbol{u}, \\
E & =\int_{\mathbb{R}^{2}} \frac{1}{2}\left(\|\boldsymbol{\zeta}\|^{2} \boldsymbol{a}_{1}+\boldsymbol{a}_{2}\right) \cdot \boldsymbol{f} d \boldsymbol{\zeta}=\frac{1}{2}\left(\frac{3}{2} \rho T+\rho\|\boldsymbol{u}\|^{2}\right) .
\end{aligned}
$$

It is considered that the stationary state has been achieved either when the iteration number of the time step reaches $10^{6}$ or when the above moments simultaneously satisfy the following conditions:

$$
\begin{aligned}
& \frac{\left\|\rho^{(n)}-\rho^{(n-1)}\right\|_{L^{2}(D)}}{\left\|\rho^{(n)}\right\|_{L^{2}(D)}}<\varepsilon_{\rho}, \\
& \frac{\left\|M^{(n)}-M^{(n-1)}\right\|_{L^{2}(D)}}{\left\|M^{(n)}\right\|_{L^{2}(D)}}<\varepsilon_{M}, \\
& \frac{\left\|E^{(n)}-E^{(n-1)}\right\|_{L^{2}(D)}}{\left\|E^{(n)}\right\|_{L^{2}(D)}}<\varepsilon_{E},
\end{aligned}
$$

where the superscript $n$ is the iteration number of the time step in the flow analysis and $\varepsilon_{\rho}, \varepsilon_{M}, \varepsilon_{E}$ are judgment criteria. On the other hand, the convergence of the adjoint flow field is judged by using three macroscopic quantities $\rho_{\mathrm{ad}}, \boldsymbol{u}_{\mathrm{ad}}$ and $T_{\mathrm{ad}}$, which appear in the collision term of adjoint equation, as follows:

$$
\begin{aligned}
& \frac{\left\|\rho_{\mathrm{ad}}^{(n)}-\rho_{\mathrm{ad}}^{(n-1)}\right\|_{L^{2}(D)}}{\left\|\rho_{\mathrm{ad}}^{(n)}\right\|_{L^{2}(D)}}<\varepsilon_{\mathrm{ad}}, \\
& \frac{\left\|\boldsymbol{u}_{\mathrm{ad}}^{(n)}-\boldsymbol{u}_{\mathrm{ad}}^{(n-1)}\right\|_{L^{2}(D)}}{\left\|\boldsymbol{u}_{\mathrm{ad}}^{(n)}\right\|_{L^{2}(D)}}<\varepsilon_{\mathrm{ad}}, \\
& \frac{\left\|T_{\mathrm{ad}}^{(n)}-T_{\mathrm{ad}}^{(n-1)}\right\|_{L^{2}(D)}}{\left\|T_{\mathrm{ad}}^{(n)}\right\|_{L^{2}(D)}}<\varepsilon_{\mathrm{ad}},
\end{aligned}
$$

\section{adjoint flow field has been achieved when the iteration number of the time step reaches $10^{6}$ or when these conditions are simultaneously satisfied.}

To save the computation time, the initial values of state variables in an optimization iteration are set as their converged values in the previous iteration since the stationary states of flows in two sequential optimization iterations can be expected to be similar, and the same is true of adjoint variables.

\section{Numerical examples}

The validity and usefulness of the proposed method are shown through several numerical examples. In the following examples, the Strouhal number Sh is set as 1. Moreover, the transition width $w$ of the normalized density is set as 1 . The initial value of $\phi$ has to be in the transition width, otherwise the design sensitivity $\mathcal{L}_{\phi}^{\prime}$ stays at 0 . The parameter $q$ for controlling the curvatures of $\boldsymbol{u}_{\gamma}$ and $T_{\gamma}$ is set as 0.1 .

\subsection{Validation of the extended BGK equation}

To show the validity of the extended BGK equation, the result of the analysis using the extended BGK equation is compared with that of the analysis explicitly using the boundary conditions on the gassolid interfaces. For the comparison, a Couette flow, that is, a gas flow between two infinite plane walls is considered. One wall at $x_{2}=0$ is at rest and keeps the temperature at $T_{w}=1$ and the other at $x_{2}=1$ moves at a constant velocity $\boldsymbol{u}_{w}=[0.01,0]^{T}$ and also keeps the temperature at $T_{w}$. From the physical point of 
view, it is found that the solution is constant along the $x_{1}$ axis, so that a finite domain $\Omega=\left\{0 \leq x_{1}, x_{2} \leq 1\right\}$ is taken as the analysis domain of the rarefied gas and the periodic boundary condition is imposed on the left and right boundaries of the domain. Whereas the lower wall is modeled as a solid domain $D \backslash \Omega$ with temperature set as $T_{w}$ in the analysis using the proposed method, the diffuse-reflection boundary condition is imposed on the lower wall in the other analysis as shown in Fig. 田. The upper wall is modeled using the diffuse-reflection boundary condition in both analyses. The gas domain $\Omega$ and solid domain $D \backslash \Omega$ are

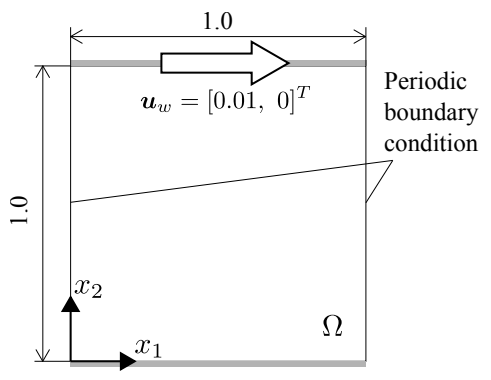

(a)

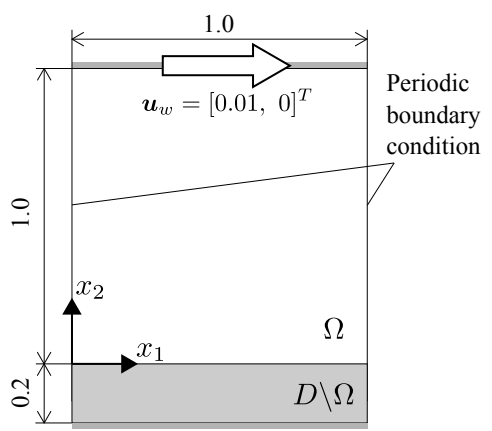

(b)

Figure 4: The analysis model (a) using the diffuse-reflection boundary condition explicitly and (b) using the proposed method.

divided into a grid of $100 \times 100$ squares and $100 \times 20$ squares, respectively. On the other hand, the finite velocity space $D_{\zeta}$ with the threshold $Z=4.5$ is discretized on a $38 \times 38$ grid. In solid domains, the Knudsen number is set as $1 \times 10^{-3}$.

Fig. 1 shows the distributions of flow velocity $u_{1}$ at $x_{1}=0.5$ in both analyses for three different Knudsen numbers: $\mathrm{Kn}=0.01,0.1$ and 1. It illustrates that the result of the analysis using the extended BGK equation coincides well with that of the analysis explicitly using the diffuse-reflection boundary condition on the lower wall. Thus, the flow state with the diffuse-reflection boundary condition imposed on the gas-solid interfaces can be approximated by using the extended BGK equation.

\subsection{Validation of sensitivity}

The validity of the derived design sensitivity $\mathcal{L}_{\phi}^{\prime}$ is confirmed through the comparison with the sensitivity computed by the finite difference method. A pressure loss minimization problem is considered here, and the objective functional is defined as

$$
J=\int_{\Theta} \int_{\Gamma_{\text {in }}} \rho T d \Gamma d t-\int_{\Theta} \int_{\Gamma_{\text {out }}} \rho T d \Gamma d t
$$

where the product of density and temperature is pressure. The fixed design domain $D$ is composed of the circular domain with $\phi=-0.8$ and the remaining domain with $\phi=0.8$ as shown in Fig. G. Since the analysis model is symmetric in the vertical direction, only the upper half of the model is computed. The computed model is divided into a grid of $100 \times 50$ squares. The finite velocity space $D_{\zeta}$ with the threshold $Z=4.5$ is discretized on a $38 \times 38$ grid. The inlet flow velocity $\overline{\boldsymbol{u}}$ is given the parabolic distribution so that its mean speed is $5.0 \times 10^{-3}$. The density is prescribed as $\bar{\rho}=1$ on the outlet, and the temperature on the boundaries is set as $T_{w}=T_{\mathrm{in}}=T_{\text {out }}=1$. The initial condition of the state variable $\boldsymbol{f}$ is set as $\boldsymbol{f}_{0}=\boldsymbol{f}^{\mathrm{eq}}\left(\bar{\rho}, \mathbf{0}, T_{w}\right)$. The temperature of the solid is set as $T_{s}=1$ and the Knudsen number in solid domains is defined as $1 \times 10^{-3}$.

The sensitivity computed by the finite difference method is defined by

$$
J_{\mathrm{FD}}^{\prime}\left(\boldsymbol{x}^{\prime}\right)=\frac{J\left[\phi+\mathbb{I}\left(\boldsymbol{x}-\boldsymbol{x}^{\prime}\right) \Delta \phi\right]-J\left[\phi-\mathbb{I}\left(\boldsymbol{x}-\boldsymbol{x}^{\prime}\right) \Delta \phi\right]}{2 \Delta \phi},
$$




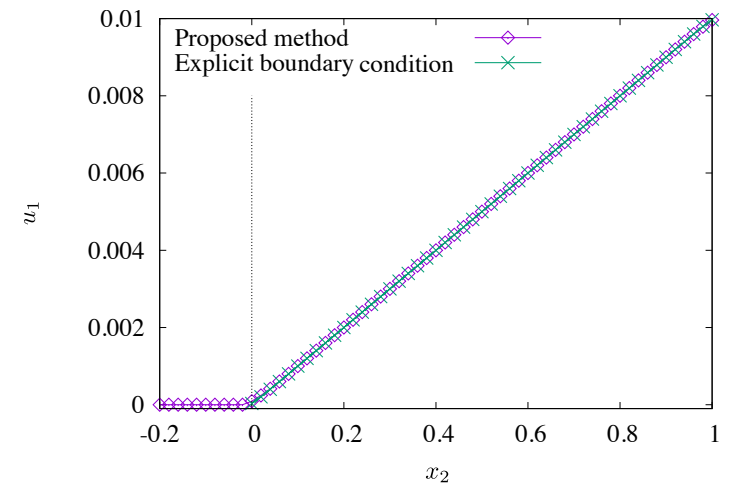

(a)

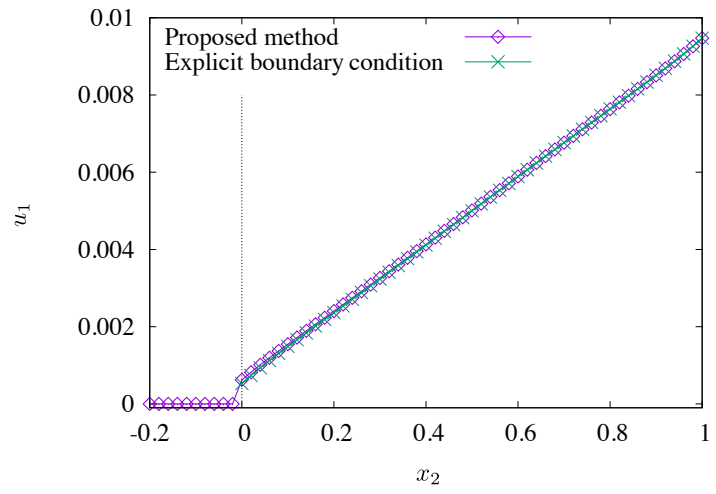

(b)

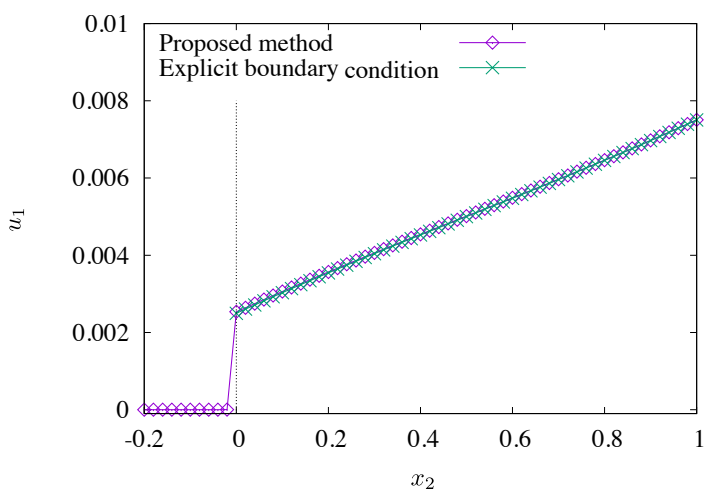

(c)

Figure 5: Comparison of the distribution of the flow velocity $u_{1}$ on $x_{1}=0.5$ for (a) Kn=0.01, (b) Kn $=0.1$ and (c) Kn $=1$.

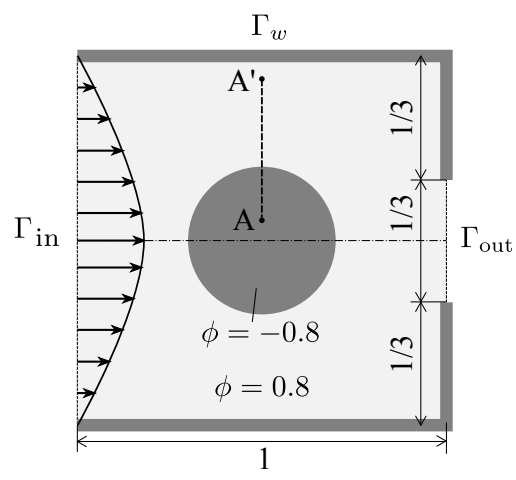

Figure 6: Analysis model for validation of sensitivity. 
where

$$
\mathbb{I}(\boldsymbol{x})=\left\{\begin{array}{lll}
1 & \text { if } & \boldsymbol{x}=\mathbf{0} \\
0 & \text { if } & \boldsymbol{x} \neq \mathbf{0}
\end{array} .\right.
$$

Both sensitivities $\mathcal{L}_{\phi}^{\prime}$ and $J_{\mathrm{FD}}^{\prime}$ with $\Delta \phi=1 \times 10^{-4}$ are compared on the line segment $\mathrm{AA}^{\prime}$ in Fig. 6 .

Fig. $\mathbb{U}$ shows the comparison results for two different Knudsen numbers of gas: $\mathrm{Kn}=0.01$ and 1 . It is noted that each sensitivity is normalized with its mean value. From these results, it can be confirmed that the derived sensitivity coincides with the sensitivity computed by the finite difference method.

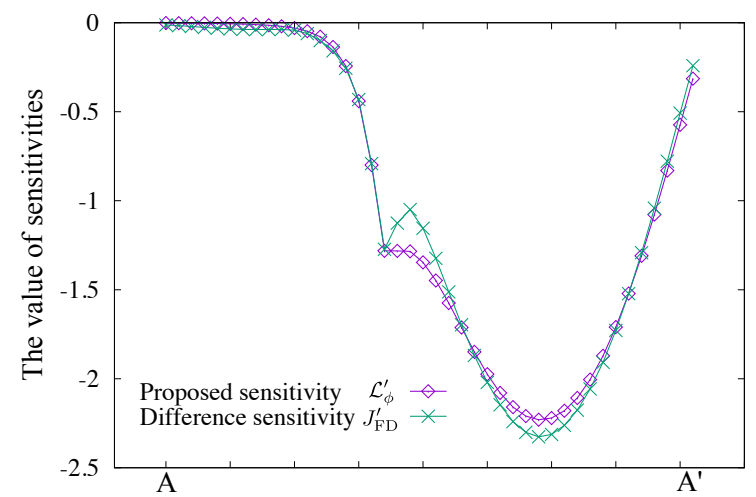

(a)

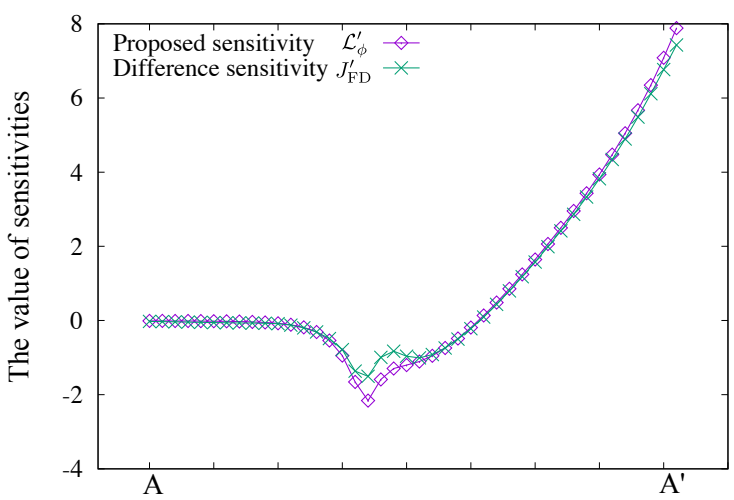

(b)

Figure 7: Comparison of the sensitivities on the line segment $\mathrm{AA}^{\prime}$ for (a) $\mathrm{Kn}=0.01$ and (b) $\mathrm{Kn}=1$.

\subsection{Bend pipe design problem}

The proposed method is applied to a bend pipe design problem as shown in Fig. $\mathbb{8}$. The objective functional is a pressure loss defined as Eq. (G0), and the volume constraint is set as $V_{\max }=0.25$. The

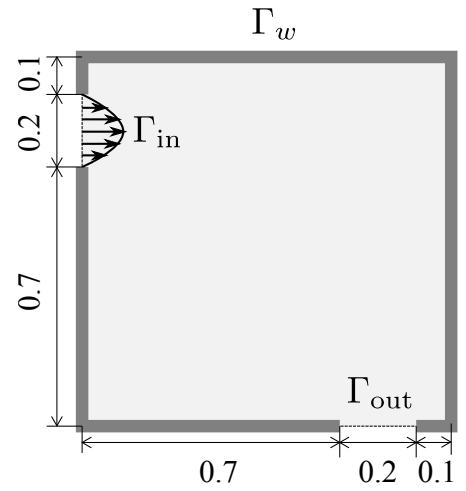

Figure 8: Domain and boundary settings of the bend pipe design problem.

fixed design domain is divided into a grid of $100 \times 100$ squares and the finite velocity space $D_{\zeta}$ with the threshold $Z=4.5$ is divided into a grid of $38 \times 38$ squares. The initial value of design variable $\phi$ is set so that $\phi=0.9$ elsewhere in the fixed design domain. As same as the problem in Sec. 5.2 , the velocity distribution is prescribed to be parabolic with the mean speed set as $5.0 \times 10^{-3}$ on the inlet, the density is prescribed as $\bar{\rho}=1$ on the outlet, and the temperature on the boundaries is set as $T_{w}=T_{\mathrm{in}}=T_{\text {out }}=1$. The initial 
condition of the state variable is set as $\boldsymbol{f}_{0}=\boldsymbol{f}^{\mathrm{eq}}\left(\bar{\rho}, \mathbf{0}, T_{w}\right)$. The temperature of the solid is set as $T_{s}=1$ and the Knudsen number in solid domains is defined as $1 \times 10^{-3}$. The convergence criterion for the objective functional is set as $\varepsilon_{\mathrm{opt}}=1 \times 10^{-5}$. With regard to the convergence of the state flow field, the criterion is set as $\varepsilon_{\rho}=\varepsilon_{E}=5.0 \times 10^{-7}$ and $\varepsilon_{M}=5.0 \times 10^{-6}$. On the other hand, the criterion for the adjoint flow field is set as $\varepsilon_{\mathrm{ad}}=5.0 \times 10^{-6}$.

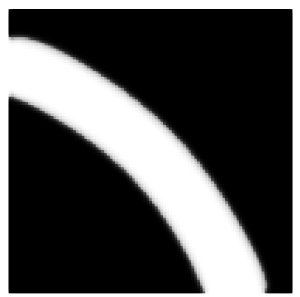

(a)

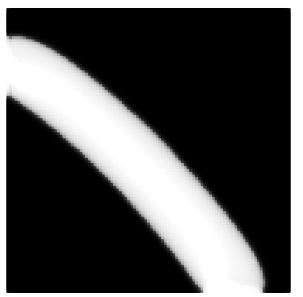

(b)

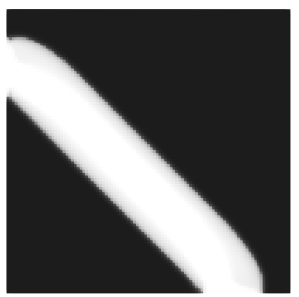

(c)

Figure 9: Optimal configurations for (a) $\mathrm{Kn}=0.01$, (b) $\mathrm{Kn}=0.1$ and (c) $\mathrm{Kn}=1$. White domains represent rarefied gas domains, while black domains represent solid domains.

Fig. $\mathbf{Q}$ shows the optimal configurations obtained through our proposed method for three different Knudsen numbers: $\mathrm{Kn}=0.01,0.1$ and 1. It illustrates that the curvature of the optimal structure is smaller as the Knudsen number is larger. The time required for these optimization calculations on a PC with 80 Intel Xeon processors at $2.20 \mathrm{GHz}$ are reported in Table $\mathrm{W}$. To confirm the validity of the obtained optimal

\begin{tabular}{c||ccc} 
Kn & 0.01 & 0.1 & 1 \\
\hline time(h) & 40.5 & 56.9 & 37.9
\end{tabular}

Table 1: Time required for the optimization calculations.

configurations, rarefied gas flows with the different Knudsen numbers at each optimal configuration were analyzed, and the values of the objective functional were compared. It is noted that each flow was recalculated after the initial value of state variables was reset to $\boldsymbol{f}_{0}$ to equalize the analysis condition. The result is shown in Table 2 .

\begin{tabular}{c||ccc}
\multicolumn{1}{c||}{} & \multicolumn{3}{c}{ Kn for optimization } \\
\hline Kn of gas & 0.01 & 0.1 & 1 \\
\hline 0.01 & $\mathbf{2 . 3 7}$ & 2.46 & 2.39 \\
0.1 & 2.90 & 2.41 & $\mathbf{2 . 4 0}$ \\
1 & 2.74 & 2.29 & $\mathbf{2 . 2 3}$
\end{tabular}

Table 2: Comparison of the values of objective functional $\left(\times 10^{-3}\right)$. The minimum value in each row is shown in bold.

From Table ¿, it can be seen that the values of the objective functional become minimum when Knudsen number of gas coincides with that for optimization, except the gas whose $\mathrm{Kn}=0.1$. However, in the case of gas whose $\mathrm{Kn}=0.1$, there is little difference between the value of the objective functional at the optimal channel for $\mathrm{Kn}=0.1$ and that at the optimal channel for $\mathrm{Kn}=1$, so that the difference is considered to be caused by numerical errors. Therefore, it can be confirmed that the appropriate optimal configuration for each gas flow is obtained by the proposed method.

\subsection{Optimum design problem of a thermally driven pump}

Next, the proposed method is applied to a thermally driven pump design problem. This structural optimization problem is replaced with the problem to find the optimal distribution of rarefied gas domains 
and solid domains with high and low temperature, with the volume limit set as $V_{\max }=0.8$. The temperature gradients in the rarefied gas domains, which are the driving force of the pump, are caused by the solids with different temperature. As shown in Fig. एు, it is assumed that the pump has a periodic structure. To design a unit of the periodic structure, the specular-reflection condition is imposed on the top and bottom boundaries of the analysis domain to model symmetry boundaries, and the periodic boundary condition is imposed on the right and left boundaries. At the center of the analysis domain, the non-design domain, that is, the domain occupied only with rarefied gas, is set along the $x_{1}$ axis. Using this domain as a divider for preventing solid domains with different temperatures from attaching to each other, the solid domains with high temperature are placed above this non-design domain, while that with low temperature are placed below the non-design domain. Further, the evaluation domain is set on the right side of the analysis domain. From the engineering view point, the ratio between the mass flow rates of thermally driven and pressure driven flows is practical as the objective functional in the design of a pump. However, it makes the numerical analysis of the flow complicated. To avoid this complexity, the mass flow rate in the desired direction,

$$
J=-\int_{\Theta} \int_{\Omega_{e}} \boldsymbol{u} \cdot \boldsymbol{e}_{1} d D d t
$$

is chosen as the simplified objective functional, where $\boldsymbol{e}_{1}$ means the unit vector along the $x_{1}$ axis. The

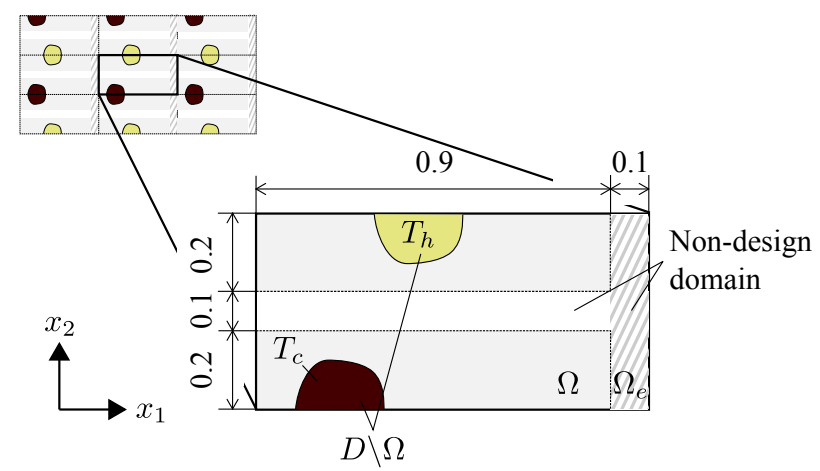

Figure 10: Domain settings.

analysis domain is divided into a grid of $100 \times 50$ squares. The finite velocity space $D_{\zeta}$ with the threshold $Z=6$ is discretized on a $62 \times 62$ grid. The initial value of design variable $\phi$ is set so that $\phi=-0.9$ in two small circular domains and $\phi=0.9$ in the remaining domain except for the evaluation domain and non-design domain, as illustrated in Fig. ㅁ]. The temperature in the solid domains is set as $T_{h}=1.5$ and

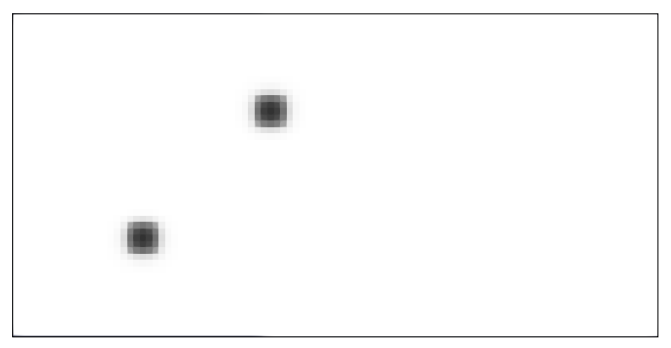

Figure 11: Initial configuration.

$T_{c}=0.5$. The initial condition of the state variable is set as $\boldsymbol{f}_{0}=\boldsymbol{f}^{\mathrm{eq}}\left(1, \mathbf{0},\left(T_{h}+T_{c}\right) / 2\right)$. The Knudsen number in gas and solid domains are set as 0.5 and $1 \times 10^{-4}$, respectively. The convergence criterion for the objective functional is set as $\varepsilon_{\mathrm{opt}}=1 \times 10^{-5}$. With regard to the convergence of the state flow field, 


\begin{tabular}{c||c|c|c} 
& Optimal configuration & Binarized optimal configuration & Reference model \\
\hline$J$ & -15.8 & -3.20 & -2.34
\end{tabular}

Table 3: Comparison of the values of objective functional $\left(\times 10^{-4}\right)$.

the criterion is set as $\varepsilon_{\rho}=\varepsilon_{E}=1.0 \times 10^{-7}$ and $\varepsilon_{M}=1.0 \times 10^{-6}$. On the other hand, the criterion for the adjoint flow field is set as $\varepsilon_{\text {ad }}=1.0 \times 10^{-6}$.

In advance of the optimization, the validity of the discretization of the finite velocity space was confirmed since thermally driven flow problems are more complex than iso-thermal flow problems. The values of the design sensitivity on the initial configuration were compared, based on the velocity discretization by different grids: uniform $62 \times 62,80 \times 80,100 \times 100$ grids, and non-uniform $62 \times 62$ grid. The non-uniform grid was constructed according to the literature [5.5], that is, the discretized velocity $\zeta_{k}\left(k=1, \ldots, N_{\zeta}\right)$ was defined as

$$
\zeta_{k}=\frac{Z}{\left(N_{\zeta}-1\right)^{\iota}}\left(2 k-N_{\zeta}-1\right)^{\iota}
$$

with the parameter $\iota=3$, where $N_{\zeta}$ is the number of grid points. Fig. Ш2 shows the distributions of the sensitivity values $\mathcal{L}_{\phi}^{\prime}$ on $x_{2}=0.1$ and $x_{2}=0.4$. From Fig. ए2, it can be found that the uniform $62 \times 62$ grid is enough for this problem since each grid provides a similar distribution of the design sensitivity.

Fig. [3] shows the optimal configuration and its velocity distribution with isothermal lines. The time required for this optimization calculation on a PC with 80 Intel Xeon processors at $2.20 \mathrm{GHz}$ was 230 hours. It should be noted that the obtained configuration has the possibility of being a local optimum due to the gradient-based optimization method and the periodicity of the design model. Nevertheless, the optimal configuration is similar to the design of the pump proposed by Sugimoto and Sone [56]. It illustrates that the positive thermal gradient with respect to the $x_{1}$ axis appears in the pure white regions, that is, rarefied gas domains, while the negative thermal gradient is steep in faintly grayish regions, namely grayscale regions. In spite of such steep negative thermal gradients with respect to the $x_{1}$ axis which enables the inducement of the strong flow in the $-\boldsymbol{e}_{1}$ direction, the gas flows in the $\boldsymbol{e}_{1}$ direction in the grayscale regions of the optimal configuration. For this reason, it can be deduced that the intermediate value of $\gamma$ in grayscale regions prevents the rarefied gas from flowing in the $-\boldsymbol{e}_{1}$ direction due to the decrease of Knudsen number and, at the same time, does not affect the flow velocity $\boldsymbol{u}_{\gamma}$ so much. In the optimal configuration, such behavior of the rarefied gas flow in grayscale regions is utilized not to decelerate the flow by the inevitable negative thermal gradient which is produced because of the periodicity of the structure. Although it implies that the better performance of the pump can be achieved if the flow is realized in those regions in practice, we here do not assume the use of any other materials than the rarefied gas and solid. Although it implies that the better performance of the pump can be achieved if the flow is realized in those regions in practice, the use of any other materials than the rarefied gas and solid is not assumed in this work.

Therefore, to show the usefulness of the obtained configuration, the performance of the binarized optimal configuration with the threshold of the normalized density $\gamma=0.5$ was examined. Fig. ए4 shows the velocity distribution with iso-thermal lines in the binarized optimal configuration. As shown in Fig. प4, the negative thermal gradient against the $x_{1}$ axis becomes gentler than positive ones, which generates the flow in the direction $\boldsymbol{e}_{1}$ on the whole.

For the comparison of the performance, a reference model with the same volume as the binarized optimal model was arranged, as shown in Fig. … The value of objective functional $J$ in each pump design is summed up as Table 3 . The value of $J$ in the binarized optimal configuration becomes worse than that in the original optimal configuration since the preferable behavior of the flow in grayscale regions can no longer be used. Nevertheless, the value of $J$ in the binarized optimal configuration is superior to that in the reference model, so that the proposed method works well for designs of thermally driven devices, although the room of improvement about how to handle grayscale regions is still left. 


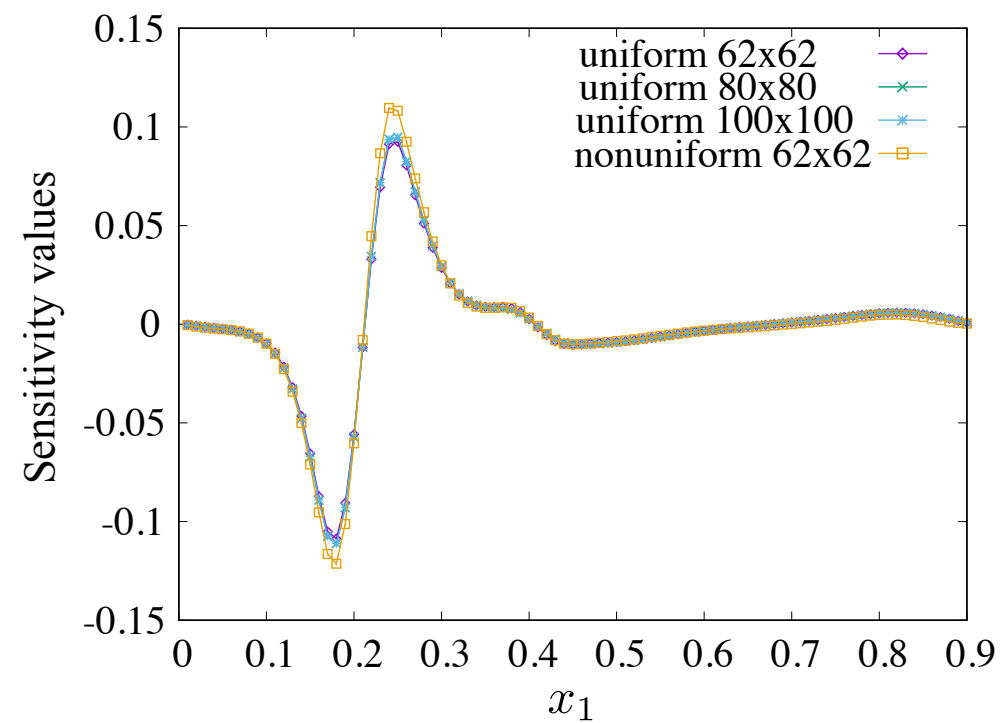

(a)

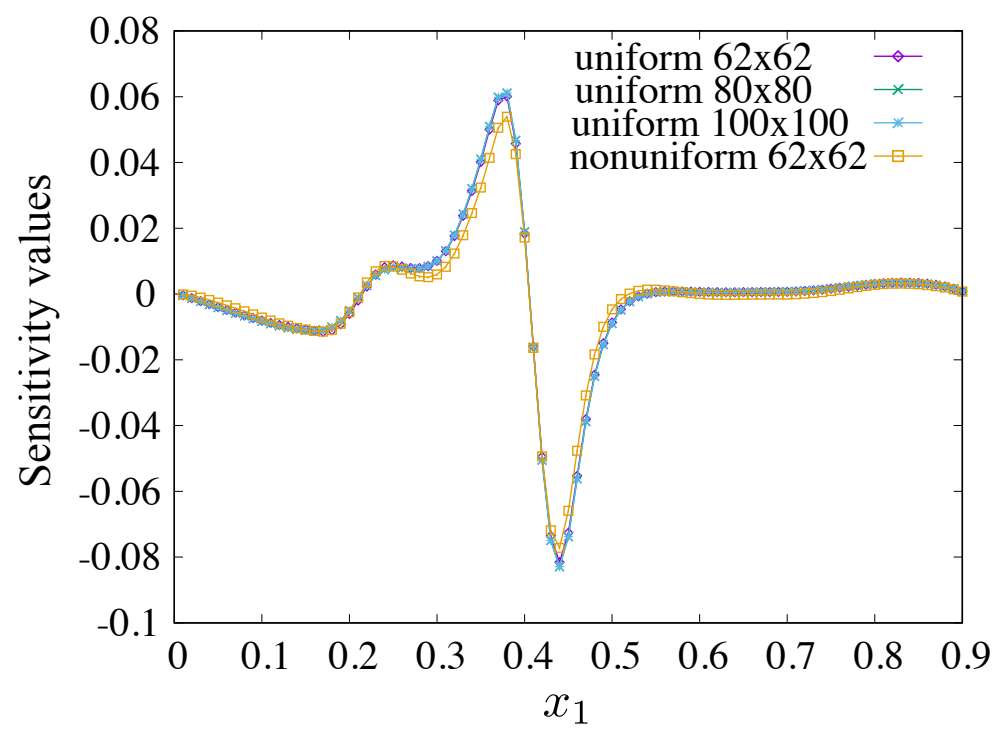

(b)

Figure 12: The sensitivity values on (a) $x_{2}=0.1$ and (b) $x_{2}=0.4$. 


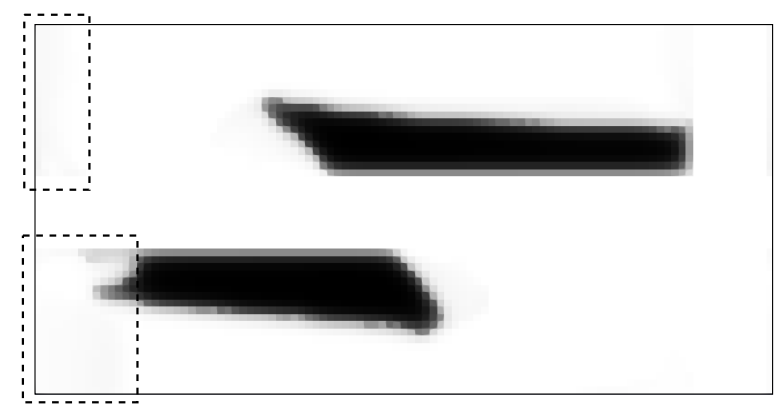

(a)

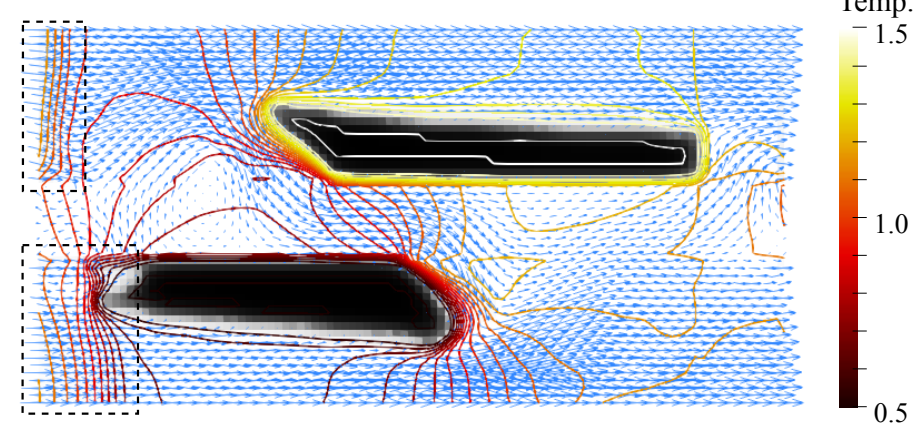

(b)

Figure 13: (a) The optimal configuration and (b) its velocity distribution with iso-thermal lines. White domains represent rarefied gas domains, while black domains represent solid domains. In the grayscale regions surrounded by black dash lines, the thermal gradient in $-\boldsymbol{e}_{1}$ direction becomes steep.

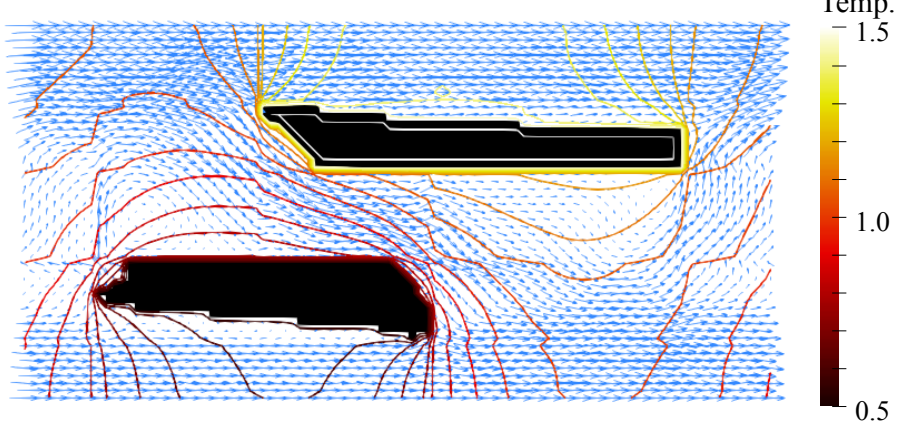

Figure 14: The velocity distribution with iso-thermal lines in the binarized optimal configuration. 


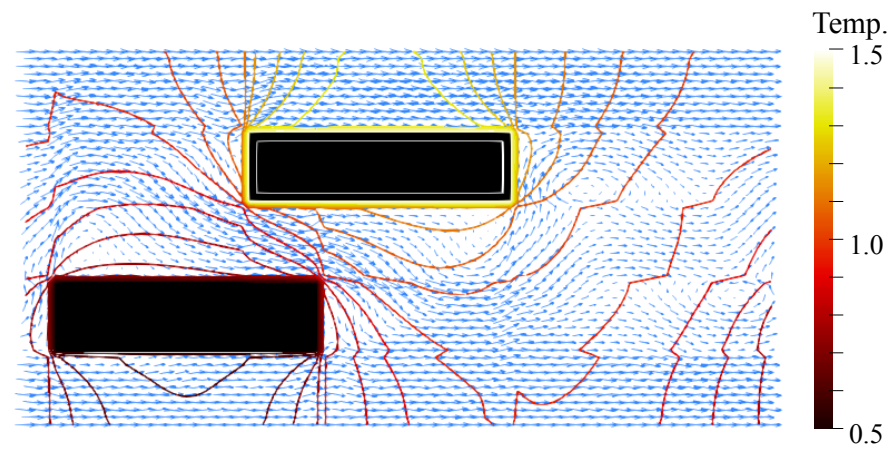

Figure 15: The velocity distribution with iso-thermal lines in the reference model.

\section{Conclusions}

This paper presented a topology optimization method in rarefied gas flow problems. The detailed achievements we obtained are the following:

1. Topology optimization problems for the design of 2-D rarefied gas flow channels were formulated. To incorporate the shape representation of topology optimization to the rarefied gas flow problems, the physical field was extended to the entire design domain including the solid domains, assuming that the solid domains were occupied by a fictitious gas with the static equilibrium state. The BGK equation, the governing equation of rarefied gas flows, was also extended based on the extension of the physical field, which enabled the flow state on the gas-solid interfaces to be approximated.

2. According to the formulation of the optimization problems, the optimization algorithm was constructed. In this study, a density method based on the Heaviside projection method with the PDE filter was adopted. The design variable, namely the scalar function $\phi$, was updated by MMA, based on the design sensitivity which was derived by the Lagrange multiplier method and adjoint variable method. As is the case of the governing equation, the adjoint equation obtained from the sensitivity analysis was computed with an explicit finite difference method after replacing the infinite molecular velocity space with the finite one.

3. Through numerical examples, the validity and utility of the proposed method were confirmed. In the bend pipe problem, the dependency of optimal configurations on the Knudsen number was shown. In the design problem of a thermally driven pump, the optimal configuration with some grayscale regions was obtained, yet the binarized configuration displayed superior performance to the reference model we arranged.

The proposed method has the potential to be applied to the design of various Micro Electro Mechanical System (MEMS) devices inside which gas flows exist, including micro-pumps. To improve the applicability of the method, future work to tackle the grayscale problem seen here would also be performed.

\section{Acknowledgements}

This work was supported by JSPS KAKENHI Grant Number 18J13839.

\section{Appendix A. Derivation of the adjoint equation}

The procedure for the derivation of the adjoint initial-boundary value problem is described in detail. At first, according to Eq. (45), the Gâteaux derivative for variable $\tilde{\boldsymbol{f}}$ is performed, using Gauss's divergence 
theorem with regard to time and space.

$$
\begin{aligned}
& d \mathcal{L}(\tilde{\boldsymbol{f}} ; \delta \tilde{\boldsymbol{f}})=\int_{D} \int_{\mathbb{R}^{2}}[\operatorname{Sh} \tilde{\boldsymbol{g}} \cdot \delta \tilde{\boldsymbol{f}}]_{t_{i}}^{t_{f}} d \boldsymbol{\zeta} d D-\int_{\Theta} \int_{\partial D} \int_{\mathbb{R}^{2}}(\boldsymbol{\zeta} \cdot \boldsymbol{n})(\tilde{\boldsymbol{g}} \cdot \delta \tilde{\boldsymbol{f}}) d \boldsymbol{\zeta} d \Gamma d t \\
& +\int_{\Theta} \int_{D} \int_{\mathbb{R}^{2}}\left(-\operatorname{Sh} \frac{\partial \tilde{\boldsymbol{g}}}{\partial t}-(\boldsymbol{\zeta} \cdot \nabla) \tilde{\boldsymbol{g}}+\frac{\tilde{\rho}}{k_{\gamma}} \tilde{\boldsymbol{g}}\right) \cdot \delta \tilde{\boldsymbol{f}} d \boldsymbol{\zeta} d D d t \\
& +\int_{D} \int_{\mathbb{R}^{2}} \tilde{\boldsymbol{g}}_{0} \cdot \delta \tilde{\boldsymbol{f}}\left(t_{i}, \boldsymbol{x}, \boldsymbol{\zeta}\right) d \boldsymbol{\zeta} d D \\
& +\int_{\Theta} \int_{\Gamma_{w}} \int_{\mathbb{R}_{+}^{2}} \tilde{\boldsymbol{g}}_{w} \cdot \delta \tilde{\boldsymbol{f}} d \boldsymbol{\zeta} d \Gamma d t \\
& +\int_{\Theta} \int_{\Gamma_{w}} \int_{\mathbb{R}_{-}^{2}} 2 \tilde{s}_{w} \sqrt{\frac{\pi}{T_{w}}}(\boldsymbol{\zeta} \cdot \boldsymbol{n})\left(\boldsymbol{a}_{1} \cdot \delta \tilde{\boldsymbol{f}}\right) d \boldsymbol{\zeta} d \Gamma d t \\
& +\int_{\Theta} \int_{\Gamma_{\text {in }}} \int_{\mathbb{R}_{+}^{2}} \tilde{\boldsymbol{g}}_{\mathrm{in}} \cdot \delta \tilde{\boldsymbol{f}} d \boldsymbol{\zeta} d \Gamma d t \\
& -\int_{\Theta} \int_{\Gamma_{\mathrm{in}}} \int_{\mathbb{R}_{-}^{2}} \frac{2 \tilde{s}_{\mathrm{in}}}{\overline{\boldsymbol{u}} \cdot \boldsymbol{n}-\sqrt{\frac{T_{\mathrm{in}}}{\pi}}}(\boldsymbol{\zeta}-\overline{\boldsymbol{u}}) \cdot \boldsymbol{n}\left(\boldsymbol{a}_{1} \cdot \delta \tilde{\boldsymbol{f}}\right) d \boldsymbol{\zeta} d \Gamma d t \\
& +\int_{\Theta} \int_{\Gamma_{\mathrm{out}}} \int_{\mathbb{R}_{+}^{2}} \tilde{\boldsymbol{g}}_{\mathrm{out}} \cdot \delta \tilde{\boldsymbol{f}} d \boldsymbol{\zeta} d \Gamma d t \\
& +\int_{\Theta} \int_{\Gamma_{\text {out }}} \int_{\mathbb{R}_{-}^{2}} 2 \tilde{s}_{\text {out }} \boldsymbol{a}_{1} \cdot \delta \tilde{\boldsymbol{f}} d \boldsymbol{\zeta} d \Gamma d t \\
& -\int_{\Theta} \int_{D} \int_{\mathbb{R}^{2}} \tilde{r}_{D}\left(\boldsymbol{a}_{1} \cdot \delta \tilde{\boldsymbol{f}}\right) d \boldsymbol{\zeta} d D d t \\
& -\int_{\Theta} \int_{D} \int_{\mathbb{R}^{2}} \frac{1}{\tilde{\rho}}\left(\tilde{\boldsymbol{v}}_{D} \cdot \boldsymbol{\zeta}\right)\left(\boldsymbol{a}_{1} \cdot \delta \tilde{\boldsymbol{f}}\right) d \boldsymbol{\zeta} d D d t \\
& -\int_{\Theta} \int_{D} \int_{\mathbb{R}^{2}} \frac{2 \tilde{\theta}_{D}}{3 \tilde{\rho}}\left(\|\boldsymbol{\zeta}-\tilde{\boldsymbol{u}}\|^{2} \boldsymbol{a}_{1}+\boldsymbol{a}_{2}\right) \cdot \delta \tilde{\boldsymbol{f}} d \boldsymbol{\zeta} d D d t \\
& -\int_{\Theta} \int_{\partial D} \int_{\mathbb{R}^{2}} \tilde{r}_{B}\left(\boldsymbol{a}_{1} \cdot \delta \tilde{\boldsymbol{f}}\right) d \boldsymbol{\zeta} d D d t \\
& -\int_{\Theta} \int_{\partial D} \int_{\mathbb{R}^{2}} \frac{1}{\tilde{\rho}}\left(\tilde{\boldsymbol{v}}_{B} \cdot \boldsymbol{\zeta}\right)\left(\boldsymbol{a}_{1} \cdot \delta \tilde{\boldsymbol{f}}\right) d \boldsymbol{\zeta} d D d t \\
& -\int_{\Theta} \int_{\partial D} \int_{\mathbb{R}^{2}} \frac{2 \tilde{\theta}_{B}}{3 \tilde{\rho}}\left(\|\boldsymbol{\zeta}-\tilde{\boldsymbol{u}}\|^{2} \boldsymbol{a}_{1}+\boldsymbol{a}_{2}\right) \cdot \delta \tilde{\boldsymbol{f}} d \boldsymbol{\zeta} d D d t
\end{aligned}
$$

$=0$. 
Since $\delta \tilde{\boldsymbol{f}}$ is an arbitrary function, the following equations are obtained:

$$
\begin{aligned}
& -\operatorname{Sh} \frac{\partial \tilde{\boldsymbol{g}}}{\partial t}-(\boldsymbol{\zeta} \cdot \nabla) \tilde{\boldsymbol{g}}+\frac{\tilde{\rho}}{k_{\gamma}} \tilde{\boldsymbol{g}}-\tilde{\boldsymbol{g}}^{\mathrm{eq}}\left(\tilde{r}_{D}, \tilde{\boldsymbol{v}}_{D}, \tilde{\theta}_{D}\right)=0 \quad \text { in } \Theta \times D \times \mathbb{R}^{2}, \\
& \operatorname{Sh} \tilde{\boldsymbol{g}}\left(t_{i}, \boldsymbol{x}, \boldsymbol{\zeta}\right)-\tilde{\boldsymbol{g}}_{0}=0 \quad \text { in } D \times \mathbb{R}^{2}, \\
& \tilde{\boldsymbol{g}}\left(t_{f}, \boldsymbol{x}, \boldsymbol{\zeta}\right)=0 \\
& (\boldsymbol{\zeta} \cdot \boldsymbol{n}) \tilde{\boldsymbol{g}}-\tilde{\boldsymbol{g}}_{w}+\tilde{\boldsymbol{g}}^{\mathrm{eq}}\left(\tilde{r}_{B}, \tilde{\boldsymbol{v}}_{B}, \tilde{\theta}_{B}\right)=0 \\
& \text { in } D \times \mathbb{R}^{2} \text {, } \\
& (\boldsymbol{\zeta} \cdot \boldsymbol{n}) \tilde{\boldsymbol{g}}-2 \tilde{s}_{w} \sqrt{\frac{\pi}{T_{w}}}(\boldsymbol{\zeta} \cdot \boldsymbol{n}) \boldsymbol{a}_{1}+\tilde{\boldsymbol{g}}^{\mathrm{eq}}\left(\tilde{r}_{B}, \tilde{\boldsymbol{v}}_{B}, \tilde{\theta}_{B}\right)=0 \quad \text { in } \Theta \times \Gamma_{w} \times \mathbb{R}_{-}^{2}, \\
& (\boldsymbol{\zeta} \cdot \boldsymbol{n}) \tilde{\boldsymbol{g}}-\tilde{\boldsymbol{g}}_{\mathrm{in}}+\tilde{\boldsymbol{g}}^{\mathrm{eq}}\left(\tilde{r}_{B}, \tilde{\boldsymbol{v}}_{B}, \tilde{\theta}_{B}\right)=0 \quad \text { in } \Theta \times \Gamma_{\text {in }} \times \mathbb{R}_{+}^{2}, \\
& (\boldsymbol{\zeta} \cdot \boldsymbol{n}) \tilde{\boldsymbol{g}}+\frac{2 \tilde{s}_{\mathrm{in}}(\boldsymbol{\zeta}-\overline{\boldsymbol{u}}) \cdot \boldsymbol{n}}{\overline{\boldsymbol{u}} \cdot \boldsymbol{n}-\sqrt{\frac{T_{\mathrm{in}}}{\pi}}} \boldsymbol{a}_{1}+\tilde{\boldsymbol{g}}^{\mathrm{eq}}\left(\tilde{r}_{B}, \tilde{\boldsymbol{v}}_{B}, \tilde{\theta}_{B}\right)=0 \quad \text { in } \Theta \times \Gamma_{\mathrm{in}} \times \mathbb{R}_{-}^{2}, \\
& (\boldsymbol{\zeta} \cdot \boldsymbol{n}) \tilde{\boldsymbol{g}}-\tilde{\boldsymbol{g}}_{\mathrm{out}}+\tilde{\boldsymbol{g}}^{\mathrm{eq}}\left(\tilde{r}_{B}, \tilde{\boldsymbol{v}}_{B}, \tilde{\theta}_{B}\right)=0 \quad \text { in } \Theta \times \Gamma_{\text {out }} \times \mathbb{R}_{+}^{2} \text {, } \\
& (\boldsymbol{\zeta} \cdot \boldsymbol{n}) \tilde{\boldsymbol{g}}-2 \tilde{s}_{\text {out }} \boldsymbol{a}_{1}+\tilde{\boldsymbol{g}}^{\mathrm{eq}}\left(\tilde{r}_{B}, \tilde{\boldsymbol{v}}_{B}, \tilde{\theta}_{B}\right)=0 \quad \text { in } \Theta \times \Gamma_{\text {out }} \times \mathbb{R}_{-}^{2},
\end{aligned}
$$

where

$$
\tilde{\boldsymbol{g}}^{\mathrm{eq}}(r, \boldsymbol{v}, \theta)=r \boldsymbol{a}_{1}+\frac{1}{\tilde{\rho}}(\boldsymbol{\zeta} \cdot \boldsymbol{v}) \boldsymbol{a}_{1}+\frac{2 \theta}{3 \tilde{\rho}}\left(\|\boldsymbol{\zeta}-\tilde{\boldsymbol{u}}\|^{2} \boldsymbol{a}_{1}+\boldsymbol{a}_{2}\right) .
$$

Likewise, Gâteaux derivatives for other variables are implemented. The Gâteaux derivative for $\tilde{\rho}$ is given by

$$
\begin{aligned}
d \mathcal{L}(\tilde{\rho} ; \delta \tilde{\rho})= & \int_{\Theta} \int_{\Omega_{e}} \frac{\partial j_{e}}{\partial \tilde{\rho}} \delta \tilde{\rho} d D d t+\int_{\Theta} \int_{\Gamma_{\mathrm{in}}} \frac{\partial j_{\mathrm{in}}}{\partial \tilde{\rho}} \delta \tilde{\rho} d \Gamma d t+\int_{\Theta} \int_{\Gamma_{\text {out }}} \frac{\partial j_{\text {out }}}{\partial \tilde{\rho}} \delta \tilde{\rho} d \Gamma d t \\
& +\int_{\Theta} \int_{D} \int_{\mathbb{R}^{2}} \tilde{\boldsymbol{g}} \cdot\left(\frac{1}{k_{\gamma}}\left(\tilde{\boldsymbol{f}}-\tilde{\boldsymbol{f}}_{\gamma}^{\mathrm{eq}}\right)-\frac{\tilde{\rho}}{k_{\gamma}} \frac{1}{\tilde{\rho}} \tilde{\boldsymbol{f}}_{\gamma}^{\mathrm{eq}}\right) \delta \tilde{\rho} d \boldsymbol{\zeta} d D d t \\
& +\int_{\Theta} \int_{D} \tilde{r}_{D} \delta \tilde{\rho} d D d t \\
& +\int_{\Theta} \int_{D} \int_{\mathbb{R}^{2}} \frac{1}{\tilde{\rho}^{2}}\left(\tilde{\boldsymbol{v}}_{D} \cdot \boldsymbol{\zeta}\right)\left(\boldsymbol{a}_{1} \cdot \tilde{\boldsymbol{f}}\right) \delta \tilde{\rho} d \boldsymbol{\zeta} d D d t \\
& +\int_{\Theta} \int_{D} \int_{\mathbb{R}^{2}} \frac{2 \tilde{\theta}_{D}}{3 \tilde{\rho}^{2}}\left(\|\boldsymbol{\zeta}-\tilde{\boldsymbol{u}}\|^{2} \boldsymbol{a}_{1}+\boldsymbol{a}_{2}\right) \cdot \tilde{\boldsymbol{f}} \delta \tilde{\rho} d \boldsymbol{\zeta} d D d t \\
& +\int_{\Theta} \int_{\partial D} \tilde{r}_{B} \delta \tilde{\rho} d D d t \\
& +\int_{\Theta} \int_{\partial D} \int_{\mathbb{R}^{2}} \frac{1}{\tilde{\rho}^{2}}\left(\tilde{\boldsymbol{v}}_{B} \cdot \boldsymbol{\zeta}\right)\left(\boldsymbol{a}_{1} \cdot \tilde{\boldsymbol{f}}\right) \delta \tilde{\rho} d \boldsymbol{\zeta} d D d t \\
& +\int_{\Theta} \int_{\partial D} \int_{\mathbb{R}^{2}} \frac{2 \tilde{\theta}_{B}}{3 \tilde{\rho}^{2}}\left(\|\boldsymbol{\zeta}-\tilde{\boldsymbol{u}}\|^{2} \boldsymbol{a}_{1}+\boldsymbol{a}_{2}\right) \cdot \tilde{\boldsymbol{f}} \delta \tilde{\rho} d \boldsymbol{\zeta} d D d t \\
= & 0,
\end{aligned}
$$


with the notation $\tilde{\boldsymbol{f}}_{\gamma}^{\mathrm{eq}}=\boldsymbol{f}^{\mathrm{eq}}\left(\tilde{\rho}, \tilde{\boldsymbol{u}}_{\gamma}, \tilde{T}_{\gamma}\right)$, which results in the following equations:

$$
\begin{aligned}
& \int_{\mathbb{R}^{2}} \frac{1}{k_{\gamma}} \tilde{\boldsymbol{g}} \cdot\left(\tilde{\boldsymbol{f}}-2 \tilde{\boldsymbol{f}}_{\gamma}^{\mathrm{eq}}\right) d \boldsymbol{\zeta}+\tilde{r}_{D}+\frac{1}{\tilde{\rho}^{2}} \int_{\mathbb{R}^{2}}\left(\tilde{\boldsymbol{v}}_{D} \cdot \boldsymbol{\zeta}\right)\left(\boldsymbol{a}_{1} \cdot \tilde{\boldsymbol{f}}\right) d \boldsymbol{\zeta} \\
& +\frac{2 \tilde{\theta}_{D}}{3 \tilde{\rho}^{2}} \int_{\mathbb{R}^{2}}\left(\|\boldsymbol{\zeta}-\tilde{\boldsymbol{u}}\|^{2} \boldsymbol{a}_{1}+\boldsymbol{a}_{2}\right) \cdot \tilde{\boldsymbol{f}} d \boldsymbol{\zeta}=0 \quad \text { in } \Theta \times D \backslash \Omega_{e}, \\
& \frac{\partial j_{e}}{\partial \tilde{\rho}}+\int_{\mathbb{R}^{2}} \frac{1}{k_{\gamma}} \tilde{\boldsymbol{g}} \cdot\left(\tilde{\boldsymbol{f}}-2 \tilde{\boldsymbol{f}}_{\gamma}^{\mathrm{eq}}\right) d \boldsymbol{\zeta}+\tilde{r}_{D}+\frac{1}{\tilde{\rho}^{2}} \int_{\mathbb{R}^{2}}\left(\tilde{\boldsymbol{v}}_{D} \cdot \boldsymbol{\zeta}\right)\left(\boldsymbol{a}_{1} \cdot \tilde{\boldsymbol{f}}\right) d \boldsymbol{\zeta} \\
& +\frac{2 \tilde{\theta}_{D}}{3 \tilde{\rho}^{2}} \int_{\mathbb{R}^{2}}\left(\|\boldsymbol{\zeta}-\tilde{\boldsymbol{u}}\|^{2} \boldsymbol{a}_{1}+\boldsymbol{a}_{2}\right) \cdot \tilde{\boldsymbol{f}} d \boldsymbol{\zeta}=0 \quad \text { in } \Theta \times \Omega_{e}, \\
& \tilde{r}_{B}+\frac{1}{\tilde{\rho}^{2}} \int_{\mathbb{R}^{2}}\left(\tilde{\boldsymbol{v}}_{B} \cdot \boldsymbol{\zeta}\right)\left(\boldsymbol{a}_{1} \cdot \tilde{\boldsymbol{f}}\right) d \boldsymbol{\zeta}+\frac{2 \tilde{\theta}_{B}}{3 \tilde{\rho}^{2}} \int_{\mathbb{R}^{2}}\left(\|\boldsymbol{\zeta}-\tilde{\boldsymbol{u}}\|^{2} \boldsymbol{a}_{1}+\boldsymbol{a}_{2}\right) \cdot \tilde{\boldsymbol{f}} d \boldsymbol{\zeta}=0 \quad \text { in } \quad \Theta \times \Gamma_{w}, \\
& \frac{\partial j_{\mathrm{in}}}{\partial \tilde{\rho}}+\tilde{r}_{B}+\frac{1}{\tilde{\rho}^{2}} \int_{\mathbb{R}^{2}}\left(\tilde{\boldsymbol{v}}_{B} \cdot \boldsymbol{\zeta}\right)\left(\boldsymbol{a}_{1} \cdot \tilde{\boldsymbol{f}}\right) d \boldsymbol{\zeta}+\frac{2 \tilde{\theta}_{B}}{3 \tilde{\rho}^{2}} \int_{\mathbb{R}^{2}}\left(\|\boldsymbol{\zeta}-\tilde{\boldsymbol{u}}\|^{2} \boldsymbol{a}_{1}+\boldsymbol{a}_{2}\right) \cdot \tilde{\boldsymbol{f}} d \boldsymbol{\zeta}=0 \quad \text { in } \quad \Theta \times \Gamma_{\mathrm{in}}, \\
& \frac{\partial j_{\text {out }}}{\partial \tilde{\rho}}+\tilde{r}_{B}+\frac{1}{\tilde{\rho}^{2}} \int_{\mathbb{R}^{2}}\left(\tilde{\boldsymbol{v}}_{B} \cdot \boldsymbol{\zeta}\right)\left(\boldsymbol{a}_{1} \cdot \tilde{\boldsymbol{f}}\right) d \boldsymbol{\zeta}+\frac{2 \tilde{\theta}_{B}}{3 \tilde{\rho}^{2}} \int_{\mathbb{R}^{2}}\left(\|\boldsymbol{\zeta}-\tilde{\boldsymbol{u}}\|^{2} \boldsymbol{a}_{1}+\boldsymbol{a}_{2}\right) \cdot \tilde{\boldsymbol{f}} d \boldsymbol{\zeta}=0 \quad \text { in } \quad \Theta \times \Gamma_{\text {out }} .
\end{aligned}
$$

The Gâteaux derivative for $\tilde{\boldsymbol{u}}$ is

$$
\begin{aligned}
d \mathcal{L}(\tilde{\boldsymbol{u}} ; \delta \tilde{\boldsymbol{u}})= & \int_{\Theta} \int_{\Omega_{e}} \frac{\partial j_{e}}{\partial \tilde{\boldsymbol{u}}} \cdot \delta \tilde{\boldsymbol{u}} d D d t+\int_{\Theta} \int_{\Gamma_{\text {in }}} \frac{\partial j_{\text {in }}}{\partial \tilde{\boldsymbol{u}}} \cdot \delta \tilde{\boldsymbol{u}} d \Gamma d t+\int_{\Theta} \int_{\Gamma_{\text {out }}} \frac{\partial j_{\text {out }}}{\partial \tilde{\boldsymbol{u}}} \cdot \delta \tilde{\boldsymbol{u}} d \Gamma d t \\
& -\int_{\Theta} \int_{D} \int_{\mathbb{R}^{2}} \frac{\tilde{\rho}}{k_{\gamma}} \tilde{\boldsymbol{g}} \cdot \tilde{\boldsymbol{f}}_{\gamma}^{\mathrm{eq}} \frac{\gamma(1+q)}{\gamma+q} \frac{2(\boldsymbol{\zeta}-\tilde{\boldsymbol{u}}) \cdot \delta \tilde{\boldsymbol{u}}}{\tilde{T}_{\gamma}} d \boldsymbol{\zeta} d D d t \\
& +\int_{\Theta} \int_{D} \tilde{\boldsymbol{v}}_{D} \cdot \delta \tilde{\boldsymbol{u}} d D d t \\
& +\int_{\Theta} \int_{D} \int_{\mathbb{R}^{2}} \frac{2 \tilde{\theta}_{D}}{3 \tilde{\rho}}(2(\boldsymbol{\zeta}-\tilde{\boldsymbol{u}}) \cdot \delta \tilde{\boldsymbol{u}})\left(\boldsymbol{a}_{1} \cdot \tilde{\boldsymbol{f}}\right) d \boldsymbol{\zeta} d D d t \\
& +\int_{\Theta} \int_{\partial D} \tilde{\boldsymbol{v}}_{B} \cdot \delta \tilde{\boldsymbol{u}} d D d t \\
& +\int_{\Theta} \int_{\partial D} \int_{\mathbb{R}^{2}} \frac{2 \tilde{\theta}_{B}}{3 \tilde{\rho}}(2(\boldsymbol{\zeta}-\tilde{\boldsymbol{u}}) \cdot \delta \tilde{\boldsymbol{u}})\left(\boldsymbol{a}_{1} \cdot \tilde{\boldsymbol{f}}\right) d \boldsymbol{\zeta} d D d t \\
= & 0 .
\end{aligned}
$$

The obtained equations are as follows:

$$
\begin{array}{lr}
-\frac{\tilde{\rho}}{k_{\gamma}} \frac{2}{\tilde{T}_{\gamma}} \frac{\gamma(1+q)}{\gamma+q} \int_{\mathbb{R}^{2}}\left(\tilde{\boldsymbol{g}} \cdot \tilde{\boldsymbol{f}}_{\gamma}^{\mathrm{eq}}\right)(\boldsymbol{\zeta}-\tilde{\boldsymbol{u}}) d \boldsymbol{\zeta}+\tilde{\boldsymbol{v}}_{D}+\int_{\mathbb{R}^{2}} \frac{4 \tilde{\theta}_{D}}{3 \tilde{\rho}}\left(\boldsymbol{a}_{1} \cdot \tilde{\boldsymbol{f}}\right)(\boldsymbol{\zeta}-\tilde{\boldsymbol{u}}) d \boldsymbol{\zeta}=0 & \text { in } \Theta \times D \backslash \Omega_{e}, \\
\frac{\partial j_{e}}{\partial \tilde{\boldsymbol{u}}}-\frac{\tilde{\rho}}{k_{\gamma}} \frac{2}{\tilde{T}_{\gamma}} \frac{\gamma(1+q)}{\gamma+q} \int_{\mathbb{R}^{2}}\left(\tilde{\boldsymbol{g}} \cdot \tilde{\boldsymbol{f}}_{\gamma}^{\mathrm{eq}}\right)(\boldsymbol{\zeta}-\tilde{\boldsymbol{u}}) d \boldsymbol{\zeta}+\tilde{\boldsymbol{v}}_{D}+\int_{\mathbb{R}^{2}} \frac{4 \tilde{\theta}_{D}}{3 \tilde{\rho}}\left(\boldsymbol{a}_{1} \cdot \tilde{\boldsymbol{f}}\right)(\boldsymbol{\zeta}-\tilde{\boldsymbol{u}}) d \boldsymbol{\zeta}=0 & \text { in } \Theta \times \Omega_{e}, \quad \text { (A.19) } \\
\tilde{\boldsymbol{v}}_{B}+\int_{\mathbb{R}^{2}} \frac{4 \tilde{\theta}_{B}}{3 \tilde{\rho}}\left(\boldsymbol{a}_{1} \cdot \tilde{\boldsymbol{f}}\right)(\boldsymbol{\zeta}-\tilde{\boldsymbol{u}}) d \boldsymbol{\zeta}=0 & \text { in } \Theta \times \Gamma_{w}, \quad \text { (A.20) } \\
\frac{\partial j_{\text {in }}}{\partial \tilde{\boldsymbol{u}}}+\tilde{\boldsymbol{v}}_{B}+\int_{\mathbb{R}^{2}} \frac{4 \tilde{\theta}_{B}}{3 \tilde{\rho}}\left(\boldsymbol{a}_{1} \cdot \tilde{\boldsymbol{f}}\right)(\boldsymbol{\zeta}-\tilde{\boldsymbol{u}}) d \boldsymbol{\zeta}=0 & \text { in } \Theta \times \Gamma_{\text {in }}, \quad \text { (A.21) } \\
\frac{\partial j_{\text {out }}}{\partial \tilde{\boldsymbol{u}}}+\tilde{\boldsymbol{v}}_{B}+\int_{\mathbb{R}^{2}} \frac{4 \tilde{\theta}_{B}}{3 \tilde{\rho}}\left(\boldsymbol{a}_{1} \cdot \tilde{\boldsymbol{f}}\right)(\boldsymbol{\zeta}-\tilde{\boldsymbol{u}}) d \boldsymbol{\zeta}=0 & \text { in } \Theta \times \Gamma_{\text {out }} \cdot(\text { A. } 22)
\end{array}
$$


The Gâteaux derivative for $\tilde{T}$ is implemented as

$$
\begin{aligned}
d \mathcal{L}(\tilde{T} ; \delta \tilde{T})= & \int_{\Theta} \int_{\Omega_{e}} \frac{\partial j_{e}}{\partial \tilde{T}} \delta \tilde{T} d D d t+\int_{\Theta} \int_{\Gamma_{\text {in }}} \frac{\partial j_{\text {in }}}{\partial \tilde{T}} \delta \tilde{T} d \Gamma d t+\int_{\Theta} \int_{\Gamma_{\text {out }}} \frac{\partial j_{\text {out }}}{\partial \tilde{T}} \delta \tilde{T} d \Gamma d t \\
& -\int_{\Theta} \int_{D} \int_{\mathbb{R}^{2}} \tilde{\boldsymbol{g}} \cdot \frac{\tilde{\rho}}{k_{\gamma}} \frac{\gamma(1+q)}{\gamma+q}\left(\tilde{\boldsymbol{f}}_{\gamma}^{\mathrm{eq}} \frac{\left\|\boldsymbol{\zeta}-\tilde{\boldsymbol{u}}_{\gamma}\right\|^{2}}{\tilde{T}_{\gamma}^{2}}-\left(\boldsymbol{a}_{1} \otimes \boldsymbol{a}_{1}\right) \tilde{\boldsymbol{f}}_{\gamma}^{\mathrm{eq}} \frac{1}{\tilde{T}_{\gamma}}\right) \delta \tilde{T} d \boldsymbol{\zeta} d D d t \\
& +\int_{\Theta} \int_{D} \tilde{\theta}_{D} \delta \tilde{T} d D d t \\
& +\int_{\Theta} \int_{\partial D} \tilde{\theta}_{B} \delta \tilde{T} d D d t \\
= & 0
\end{aligned}
$$

and the following equations are derived:

$$
\begin{array}{lr}
-\frac{\tilde{\rho}}{k_{\gamma}} \frac{1}{\tilde{T}_{\gamma}} \frac{\gamma(1+q)}{\gamma+q} \int_{\mathbb{R}^{2}} \tilde{\boldsymbol{g}} \cdot\left(\tilde{\boldsymbol{f}}_{\gamma}^{\mathrm{eq}} \frac{\left\|\boldsymbol{\zeta}-\tilde{\boldsymbol{u}}_{\gamma}\right\|^{2}}{\tilde{T}_{\gamma}}-\left(\boldsymbol{a}_{1} \otimes \boldsymbol{a}_{1}\right) \tilde{\boldsymbol{f}}_{\gamma}^{\mathrm{eq}}\right) d \boldsymbol{\zeta}+\tilde{\theta}_{D}=0 & \text { in } \Theta \times D \backslash \Omega_{e}, \\
\frac{\partial j_{e}}{\partial \tilde{T}}-\frac{\tilde{\rho}}{k_{\gamma}} \frac{1}{\tilde{T}_{\gamma}} \frac{\gamma(1+q)}{\gamma+q} \int_{\mathbb{R}^{2}} \tilde{\boldsymbol{g}} \cdot\left(\tilde{\boldsymbol{f}}_{\gamma}^{\mathrm{eq}} \frac{\left\|\boldsymbol{\zeta}-\tilde{\boldsymbol{u}}_{\gamma}\right\|^{2}}{\tilde{T}_{\gamma}}-\left(\boldsymbol{a}_{1} \otimes \boldsymbol{a}_{1}\right) \tilde{\boldsymbol{f}}_{\gamma}^{\mathrm{eq}}\right) d \boldsymbol{\zeta}+\tilde{\theta}_{D}=0 & \text { in } \Theta \times \Omega_{e}, \\
\tilde{\theta}_{B}=0 & \text { in } \Theta \times \Gamma_{w}, \\
\frac{\partial j_{\text {in }}}{\partial \tilde{T}}+\tilde{\theta}_{B}=0 & \text { in } \Theta \times \Gamma_{\text {in }}, \\
\frac{\partial j_{\text {out }}}{\partial \tilde{T}}+\tilde{\theta}_{B}=0 & \text { in } \Theta \times \Gamma_{\text {out }} .
\end{array}
$$

For $\tilde{\sigma}_{w}, \tilde{\sigma}_{\text {in }}$ and $\tilde{\sigma}_{\text {out }}$, the following Gâteaux derivative is implemented, respectively:

$$
\begin{aligned}
& d \mathcal{L}\left(\tilde{\sigma}_{w} ; \delta \tilde{\sigma}_{w}\right)=-\int_{\Theta} \int_{\Gamma_{w}} \int_{\mathbb{R}_{+}^{2}} \tilde{\boldsymbol{g}}_{w} \cdot \frac{1}{\tilde{\sigma}_{w}} \boldsymbol{f}^{\mathrm{eq}}\left(\tilde{\sigma}_{w}, \mathbf{0}, T_{w}\right) \delta \tilde{\sigma}_{w} d \boldsymbol{\zeta} d \Gamma d t \\
&+\int_{\Theta} \int_{\Gamma_{w}} \tilde{s}_{w} \delta \tilde{\sigma}_{w} d \Gamma d t \\
&= 0, \\
& d \mathcal{L}\left(\tilde{\sigma}_{\text {in }} ; \delta \tilde{\sigma}_{\text {in }}\right)=-\int_{\Theta} \int_{\Gamma_{\text {in }}} \int_{\mathbb{R}_{+}^{2}} \tilde{\boldsymbol{g}}_{\text {in }} \cdot \frac{1}{\tilde{\sigma}_{\text {in }}} \boldsymbol{f}^{\mathrm{eq}}\left(\tilde{\sigma}_{\text {in }}, \mathbf{0}, T_{\text {in }}\right) \delta \tilde{\sigma}_{\text {in }} d \boldsymbol{\zeta} d \Gamma d t \\
&+\int_{\Theta} \int_{\Gamma_{\text {in }}} \tilde{s}_{\text {in }} \delta \tilde{\sigma}_{\text {in }} d \Gamma d t \\
&=\left.0, \int_{\text {out }} ; \delta \tilde{\sigma}_{\text {out }}\right)=- \\
& d \mathcal{L}\left(\tilde{\sigma}_{\text {out }} \int_{\mathbb{R}_{+}^{2}} \tilde{\boldsymbol{g}}_{\text {out }} \cdot \frac{1}{\tilde{\sigma}_{\text {out }}} \boldsymbol{f}^{\text {eq }}\left(\tilde{\sigma}_{\text {out }}, \mathbf{0}, T_{\text {out }}\right) \delta \tilde{\sigma}_{\text {out }} d \boldsymbol{\zeta} d \Gamma d t\right. \\
&+\int_{\Theta} \int_{\Gamma_{\text {out }}} \delta \tilde{\sigma}_{\text {out }} d \Gamma d t \\
&=0,
\end{aligned}
$$


and the following equations are obtained:

$$
\begin{array}{ll}
-\frac{1}{\tilde{\sigma}_{w}} \int_{\mathbb{R}_{+}^{2}} \tilde{\boldsymbol{g}}_{w} \cdot \boldsymbol{f}^{\mathrm{eq}}\left(\tilde{\sigma}_{w}, \mathbf{0}, T_{w}\right) d \boldsymbol{\zeta}+\tilde{s}_{w}=0 & \text { in } \Theta \times \Gamma_{w}, \\
-\frac{1}{\tilde{\sigma}_{\text {in }}} \int_{\mathbb{R}_{+}^{2}} \tilde{\boldsymbol{g}}_{\text {in }} \cdot \boldsymbol{f}^{\mathrm{eq}}\left(\tilde{\sigma}_{\text {in }}, \mathbf{0}, T_{\mathrm{in}}\right) d \boldsymbol{\zeta}+\tilde{s}_{\mathrm{in}}=0 & \text { in } \Theta \times \Gamma_{\mathrm{in}}, \\
-\frac{1}{\tilde{\sigma}_{\text {out }}} \int_{\mathbb{R}_{+}^{2}} \tilde{\boldsymbol{g}}_{\text {out }} \cdot \boldsymbol{f}^{\mathrm{eq}}\left(\tilde{\sigma}_{\text {out }}, \mathbf{0}, T_{\text {out }}\right) d \boldsymbol{\zeta}+\tilde{s}_{\text {out }}=0 & \text { in } \Theta \times \Gamma_{\text {out }} .
\end{array}
$$

Next, using the above relations, the adjoint initial-boundary value problem is obtained. In the following, it is supposed that the optimal conditions for Lagrange multipliers (44) have been already satisfied, which means that $\forall \tilde{\psi}_{\text {st }} \in \tilde{\Psi}_{\text {st }}, \tilde{\psi}_{\text {st }}=\psi_{\text {st }}$ in the Lagrangian $\mathcal{L}$.

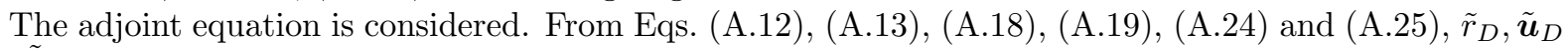
and $\tilde{\theta}_{D}$ are derived as follows:

$$
\begin{aligned}
& \tilde{r}_{D}=\left\{\begin{array}{ll}
-\int_{\mathbb{R}^{2}} \frac{1}{k_{\gamma}} \tilde{\boldsymbol{g}} \cdot\left(\boldsymbol{f}-2 \boldsymbol{f}_{\gamma}^{\mathrm{eq}}\right) d \boldsymbol{\zeta}-\frac{1}{\rho}\left(\tilde{\boldsymbol{v}}_{D} \cdot \boldsymbol{u}\right)-\frac{1}{\rho} \tilde{\theta}_{D} T & \text { in } \quad \Theta \times D \backslash \Omega_{e}, \\
-\int_{\mathbb{R}^{2}} \frac{1}{k_{\gamma}} \tilde{\boldsymbol{g}} \cdot\left(\boldsymbol{f}-2 \boldsymbol{f}_{\gamma}^{\mathrm{eq}}\right) d \boldsymbol{\zeta}-\frac{1}{\rho}\left(\tilde{\boldsymbol{v}}_{D} \cdot \boldsymbol{u}\right)-\frac{1}{\rho} \tilde{\theta}_{D} T-\frac{\partial j_{e}}{\partial \rho} & \text { in } \quad \Theta \times \Omega_{e}
\end{array},\right. \\
& \tilde{\boldsymbol{v}}_{D}=\left\{\begin{array}{ll}
\frac{\rho}{k_{\gamma}} \frac{2}{T_{\gamma}} \frac{\gamma(1+q)}{\gamma+q} \int_{\mathbb{R}^{2}}\left(\tilde{\boldsymbol{g}} \cdot \boldsymbol{f}_{\gamma}^{\mathrm{eq}}\right)(\boldsymbol{\zeta}-\boldsymbol{u}) d \boldsymbol{\zeta} & \text { in } \Theta \times D \backslash \Omega_{e}, \\
\frac{\rho}{k_{\gamma}} \frac{2}{T_{\gamma}} \frac{\gamma(1+q)}{\gamma+q} \int_{\mathbb{R}^{2}}\left(\tilde{\boldsymbol{g}} \cdot \boldsymbol{f}_{\gamma}^{\text {eq }}\right)(\boldsymbol{\zeta}-\boldsymbol{u}) d \boldsymbol{\zeta}-\frac{\partial j_{e}}{\partial \boldsymbol{u}} & \text { in } \Theta \times \Omega_{e},
\end{array},\right. \\
& \tilde{\theta}_{D}= \begin{cases}\frac{\rho}{k_{\gamma}} \frac{1}{T_{\gamma}} \frac{\gamma(1+q)}{\gamma+q} \int_{\mathbb{R}^{2}} \tilde{\boldsymbol{g}} \cdot\left(\boldsymbol{f}_{\gamma}^{\mathrm{eq}} \frac{\left\|\boldsymbol{\zeta}-\tilde{\boldsymbol{u}_{\gamma}}\right\|^{2}}{T_{\gamma}}-\left(\boldsymbol{a}_{1} \otimes \boldsymbol{a}_{1}\right) \boldsymbol{f}_{\gamma}^{\mathrm{eq}}\right) d \boldsymbol{\zeta} & \text { in } \Theta \times D \backslash \Omega_{e} \\
\frac{\rho}{k_{\gamma}} \frac{1}{T_{\gamma}} \frac{\gamma(1+q)}{\gamma+q} \int_{\mathbb{R}^{2}} \tilde{\boldsymbol{g}} \cdot\left(\boldsymbol{f}_{\gamma}^{\mathrm{eq}} \frac{\left\|\boldsymbol{\zeta}-\tilde{\boldsymbol{u}}_{\gamma}\right\|^{2}}{T_{\gamma}}-\left(\boldsymbol{a}_{1} \otimes \boldsymbol{a}_{1}\right) \boldsymbol{f}_{\gamma}^{\mathrm{eq}}\right) d \boldsymbol{\zeta}-\frac{\partial j_{e}}{\partial T} & \text { in } \quad \Theta \times \Omega_{e} .\end{cases}
\end{aligned}
$$

Putting $\tilde{\rho}_{\text {ad }}=\left(k_{\gamma} / \rho\right) \tilde{r}_{D}, \tilde{\boldsymbol{u}}_{\text {ad }}=\left(k_{\gamma} / \rho\right) \tilde{\boldsymbol{v}}_{D}$ and $\tilde{T}_{\mathrm{ad}}=\left(k_{\gamma} / \rho\right) \tilde{\theta}_{D}$ and then substituting them into Eq. (स.2), the following adjoint equation is derived:

$$
-\operatorname{Sh} \frac{\partial \tilde{\boldsymbol{g}}}{\partial t}-(\boldsymbol{\zeta} \cdot \nabla) \tilde{\boldsymbol{g}}=-\frac{\rho}{k_{\gamma}}\left(\tilde{\boldsymbol{g}}-\boldsymbol{g}^{\mathrm{eq}}\left(\tilde{\rho}_{\mathrm{ad}}, \tilde{\boldsymbol{u}}_{\mathrm{ad}}, \tilde{T}_{\mathrm{ad}}\right)\right) \quad \text { in } \quad \Theta \times D \times \mathbb{R}^{2} .
$$

The initial condition of the adjoint problem is Eq. (A.4) itself.

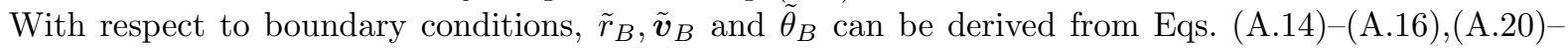
$(\overline{A .22})$ and $(\overline{A .26})-(\mathbb{A . 2 8})$ as follows:

$$
\begin{aligned}
& \tilde{r}_{B}=\left\{\begin{array}{ll}
0 & \text { in } \Theta \times \Gamma_{w} \\
-\frac{\partial j_{\text {in }}}{\partial \rho}-\frac{1}{\rho}\left(\tilde{\boldsymbol{v}}_{B} \cdot \boldsymbol{u}\right)-\frac{1}{\rho} \tilde{\theta}_{B} T & \text { in } \Theta \times \Gamma_{\text {in }} \\
-\frac{\partial j_{\text {out }}}{\partial \rho}-\frac{1}{\rho}\left(\tilde{\boldsymbol{v}}_{B} \cdot \boldsymbol{u}\right)-\frac{1}{\rho} \tilde{\theta}_{B} T & \text { in } \Theta \times \Gamma_{\text {out }}
\end{array},\right. \\
& \tilde{\boldsymbol{v}}_{B}=\left\{\begin{array}{ll}
0 & \text { in } \Theta \times \Gamma_{w} \\
-\frac{\partial j_{\text {in }}}{\partial \boldsymbol{u}} & \text { in } \Theta \times \Gamma_{\text {in }} \\
-\frac{\partial j_{\text {out }}}{\partial \boldsymbol{u}} & \text { in } \Theta \times \Gamma_{\text {out }}
\end{array},\right. \\
& \tilde{\theta}_{B}=\left\{\begin{array}{ll}
0 & \text { in } \Theta \times \Gamma_{w} \\
-\frac{\partial j_{\text {in }}}{\partial T} & \text { in } \Theta \times \Gamma_{\text {in }} \\
-\frac{\partial j_{\text {out }}}{\partial T} & \text { in } \Theta \times \Gamma_{\text {out }}
\end{array} .\right.
\end{aligned}
$$

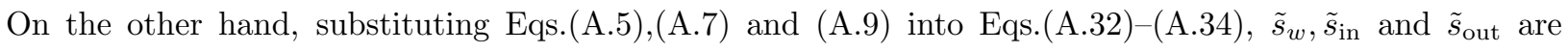


obtained as follows:

$$
\begin{aligned}
\tilde{s}_{w}=\frac{1}{\sigma_{w}} \int_{\mathbb{R}_{+}^{2}}\left((\boldsymbol{\zeta} \cdot \boldsymbol{n}) \tilde{\boldsymbol{g}}+\boldsymbol{g}^{\mathrm{eq}}\left(\tilde{r}_{B}, \tilde{\boldsymbol{v}}_{B}, \tilde{\theta}_{B}\right)\right) \cdot \boldsymbol{f}^{\mathrm{eq}}\left(\sigma_{w}, \mathbf{0}, T_{w}\right) d \boldsymbol{\zeta} & \text { in } \Theta \times \Gamma_{w}, \\
\tilde{s}_{\mathrm{in}}=\frac{1}{\sigma_{\mathrm{in}}} \int_{\mathbb{R}_{+}^{2}}\left((\boldsymbol{\zeta} \cdot \boldsymbol{n}) \tilde{\boldsymbol{g}}+\boldsymbol{g}^{\mathrm{eq}}\left(\tilde{r}_{B}, \tilde{\boldsymbol{v}}_{B}, \tilde{\theta}_{B}\right)\right) \cdot \boldsymbol{f}^{\mathrm{eq}}\left(\sigma_{\mathrm{in}}, \mathbf{0}, T_{\mathrm{in}}\right) d \boldsymbol{\zeta} & \text { in } \Theta \times \Gamma_{\text {in }}, \\
\tilde{s}_{\text {out }}=\frac{1}{\sigma_{\text {out }}} \int_{\mathbb{R}_{+}^{2}}\left((\boldsymbol{\zeta} \cdot \boldsymbol{n}) \tilde{\boldsymbol{g}}+\boldsymbol{g}^{\mathrm{eq}}\left(\tilde{r}_{B}, \tilde{\boldsymbol{v}}_{B}, \tilde{\theta}_{B}\right)\right) \cdot \boldsymbol{f}^{\mathrm{eq}}\left(\sigma_{\text {out }}, \mathbf{0}, T_{\text {out }}\right) d \boldsymbol{\zeta} & \text { in } \Theta \times \Gamma_{\text {out }} .
\end{aligned}
$$

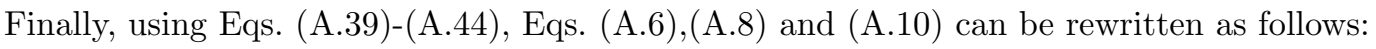

$$
\begin{aligned}
& (\boldsymbol{\zeta} \cdot \boldsymbol{n}) \tilde{\boldsymbol{g}}=2 \tilde{s}_{w} \sqrt{\frac{\pi}{T_{w}}}(\boldsymbol{\zeta} \cdot \boldsymbol{n}) \boldsymbol{a}_{1} \quad \text { in } \Theta \times \Gamma_{w} \times \mathbb{R}_{-}^{2}, \\
& (\boldsymbol{\zeta} \cdot \boldsymbol{n}) \tilde{\boldsymbol{g}}=-\frac{2 \tilde{s}_{\mathrm{in}}(\boldsymbol{\zeta}-\overline{\boldsymbol{u}}) \cdot \boldsymbol{n}}{\overline{\boldsymbol{u}} \cdot \boldsymbol{n}-\sqrt{T_{\mathrm{in}} / \pi}} \boldsymbol{a}_{1}-\boldsymbol{g}^{\mathrm{eq}}\left(\tilde{r}_{B}, \tilde{\boldsymbol{v}}_{B}, \tilde{\theta}_{B}\right) \quad \text { in } \quad \Theta \times \Gamma_{\mathrm{in}} \times \mathbb{R}_{-}^{2}, \\
& (\boldsymbol{\zeta} \cdot \boldsymbol{n}) \tilde{\boldsymbol{g}}=2 \tilde{s}_{\text {out }} \boldsymbol{a}_{1}-\boldsymbol{g}^{\mathrm{eq}}\left(\tilde{r}_{B}, \tilde{\boldsymbol{v}}_{B}, \tilde{\theta}_{B}\right) \quad \text { in } \Theta \times \Gamma_{\text {out }} \times \mathbb{R}_{-}^{2},
\end{aligned}
$$

which result in the boundary conditions in the adjoint problem.

\section{References}

[1] S. E. Vargo, E. Muntz, Initial results from the first MEMS fabricated thermal transpiration-driven vacuum pump, in: AIP Conference Proceedings, Vol. 585, AIP, 2001, pp. 502-509.

[2] S. McNamara, Y. B. Gianchandani, On-chip vacuum generated by a micromachined Knudsen pump, Journal of Microelectromechanical Systems 14 (4) (2005) 741-746.

[3] S. Takata, H. Sugimoto, S. Kosuge, Gas separation by means of the Knudsen compressor, European Journal of MechanicsB/Fluids 26 (2) (2007) 155-181.

[4] A. Passian, R. Warmack, T. Ferrell, T. Thundat, Thermal transpiration at the microscale: a Crookes cantilever, Physical Review Letters 90 (12) (2003) 124503.

[5] A. Strongrich, A. Alexeenko, Microstructure actuation and gas sensing by the Knudsen thermal force, Applied Physics Letters 107 (19) (2015) 193508

[6] W. Prager, A note on discretized Michell structures, Computer Methods in Applied Mechanics and Engineering 3 (3) (1974) 349-355.

[7] K. Svanberg, Optimization of geometry in truss design, Computer Methods in Applied Mechanics and Engineering 28 (1) (1981) 63-80.

[8] J. Sokolowski, J.-P. Zolesio, Introduction to shape optimization, in: Introduction to Shape Optimization, Springer, 1992, pp. $5-12$.

[9] B. Mohammadi, O. Pironneau, Shape optimization in fluid mechanics, Annual Review of Fluid Mechanics 36 (2004) $255-279$.

[10] M. P. Bendsøe, N. Kikuchi, Generating optimal topologies in structural design using a homogenization method, Computer Methods in Applied Mechanics and Engineering 71 (2) (1988) 197-224.

[11] G. Allaire, E. Bonnetier, G. Francfort, F. Jouve, Shape optimization by the homogenization method, Numerische Mathematik 76 (1) (1997) 27-68.

[12] M. P. Bendsøe, Optimal shape design as a material distribution problem, Structural and Multidisciplinary Optimization 1 (4) (1989) 193-202.

[13] M. P. Bendsøe, O. Sigmund, Material interpolation schemes in topology optimization, Archive of Applied Mechanics 69 (9) (1999) 635-654.

[14] M. Y. Wang, X. Wang, D. Guo, A level set method for structural topology optimization, Computer Methods in Applied Mechanics and Engineering 192 (1) (2003) 227-246.

[15] G. Allaire, F. Jouve, A.-M. Toader, Structural optimization using sensitivity analysis and a level-set method, Journal of Computational Physics 194 (1) (2004) 363-393.

[16] T. Yamada, K. Izui, S. Nishiwaki, A. Takezawa, A topology optimization method based on the level set method incorporating a fictitious interface energy, Computer Methods in Applied Mechanics and Engineering 199 (45) (2010) $2876-2891$.

[17] N. L. Pedersen, Maximization of eigenvalues using topology optimization, Structural and Multidisciplinary Optimization 20 (1) (2000) 2-11.

[18] Z.-D. Ma, N. Kikuchi, H.-C. Cheng, Topological design for vibrating structures, Computer Methods in Applied Mechanics and Engineering 121 (1-4) (1995) 259-280.

[19] S. Nishiwaki, M. I. Frecker, S. Min, N. Kikuchi, Topology optimization of compliant mechanisms using the homogenization method, International Journal for Numerical Methods in Engineering 42 (3) (1998) 535-559. 
[20] U. D. Larsen, O. Signund, S. Bouwsta, Design and fabrication of compliant micromechanisms and structures with negative Poisson's ratio, Journal of Microelectromechanical Systems 6 (2) (1997) 99-106.

[21] A. Gersborg-Hansen, M. P. Bendsøe, O. Sigmund, Topology optimization of heat conduction problems using the finite volume method, Structural and Multidisciplinary Optimization 31 (4) (2006) 251-259.

22] A. Iga, S. Nishiwaki, K. Izui, M. Yoshimura, Topology optimization for thermal conductors considering design-dependent effects, including heat conduction and convection, International Journal of Heat and Mass Transfer 52 (11) (2009) 27212732 .

[23] O. Sigmund, J. Søndergaard Jensen, Systematic design of phononic band-gap materials and structures by topology optimization, Philosophical Transactions of the Royal Society of London A: Mathematical, Physical and Engineering Sciences 361 (1806) (2003) 1001-1019.

[24] T. Borrvall, J. Petersson, Topology optimization of fluids in Stokes flow, International Journal for Numerical Methods in Fluids 41 (1) (2003) 77-107.

[25] A. Gersborg-Hansen, O. Sigmund, R. B. Haber, Topology optimization of channel flow problems, Structural and Multidisciplinary Optimization 30 (3) (2005) 181-192.

26] L. H. Olesen, F. Okkels, H. Bruus, A high-level programming-language implementation of topology optimization applied to steady-state Navier-Stokes flow, International Journal for Numerical Methods in Engineering 65 (7) (2006) 975-1001.

[27] Y. Deng, Z. Liu, P. Zhang, Y. Liu, Y. Wu, Topology optimization of unsteady incompressible Navier-Stokes flows, Journal of Computational Physics 230 (17) (2011) 6688-6708.

[28] C. S. Andreasen, A. R. Gersborg, O. Sigmund, Topology optimization of microfluidic mixers, International Journal for Numerical Methods in Fluids 61 (5) (2009) 498-513.

[29] Z. Liu, Q. Gao, P. Zhang, M. Xuan, Y. Wu, Topology optimization of fluid channels with flow rate equality constraints, Structural and Multidisciplinary Optimization 44 (1) (2011) 31-37.

[30] A. A. Koga, E. C. C. Lopes, H. F. V. Nova, C. R. de Lima, E. C. N. Silva, Development of heat sink device by using topology optimization, International Journal of Heat and Mass Transfer 64 (2013) 759-772.

31] G. H. Yoon, Topology optimization for stationary fluid-structure interaction problems using a new monolithic formulation, International Journal for Numerical Methods in Engineering 82 (5) (2010) 591-616.

[32] M. M. Gregersen, F. Okkels, M. Z. Bazant, H. Bruus, Topology and shape optimization of induced-charge electro-osmotic micropumps, New Journal of Physics 11 (7) (2009) 075019.

[33] T. Matsumori, T. Kondoh, A. Kawamoto, T. Nomura, Topology optimization for fluid-thermal interaction problems under constant input power, Structural and Multidisciplinary Optimization 47 (4) (2013) 571-581.

[34] G. H. Yoon, Topological layout design of electro-fluid-thermal-compliant actuator, Computer Methods in Applied Mechanics and Engineering 209 (2012) 28-44.

[35] V. J. Challis, J. K. Guest, Level set topology optimization of fluids in Stokes flow, International Journal for Numerical Methods in Engineering 79 (10) (2009) 1284-1308.

36] S. Zhou, Q. Li, A variational level set method for the topology optimization of steady-state Navier-Stokes flow, Journal of Computational Physics 227 (24) (2008) 10178-10195.

[37] X.-B. Duan, Y.-C. Ma, R. Zhang, Shape-topology optimization for Navier-Stokes problem using variational level set method, Journal of Computational and Applied Mathematics 222 (2) (2008) 487-499.

[38] Y. Deng, P. Zhang, Y. Liu, Y. Wu, Z. Liu, Optimization of unsteady incompressible Navier-Stokes flows using variational level set method, International Journal for Numerical Methods in Fluids 71 (12) (2013) 1475-1493.

[39] C. Othmer, A continuous adjoint formulation for the computation of topological and surface sensitivities of ducted flows, International Journal for Numerical Methods in Fluids 58 (8) (2008) 861-877.

[40] T. Inamuro, M. Yoshino, F. Ogino, Accuracy of the lattice Boltzmann method for small Knudsen number with finite Reynolds number, Physics of Fluids 9 (11) (1997) 3535-3542.

375 [41] S. Chen, G. D. Doolen, Lattice Boltzmann method for fluid flows, Annual Review of Fluid Mechanics 30 (1) (1998) 329-364.

[42] C. K. Aidun, J. R. Clausen, Lattice-Boltzmann method for complex flows, Annual Review of Fluid Mechanics 42 (2010) 439-472.

[43] G. Pingen, A. Evgrafov, K. Maute, Topology optimization of flow domains using the lattice Boltzmann method, Structural and Multidisciplinary Optimization 34 (6) (2007) 507-524.

[44] S. Kreissl, G. Pingen, A. Evgrafov, K. Maute, Topology optimization of flexible micro-fluidic devices, Structural and Multidisciplinary Optimization 42 (4) (2010) 495-516.

[45] D. Makhija, G. Pingen, R. Yang, K. Maute, Topology optimization of multi-component flows using a multi-relaxation time lattice Boltzmann method, Computers \& Fluids 67 (2012) 104-114.

6] K. Yaji, T. Yamada, M. Yoshino, T. Matsumoto, K. Izui, S. Nishiwaki, Topology optimization using the lattice Boltzmann method incorporating level set boundary expressions, Journal of Computational Physics 274 (2014) 158-181.

[47] K. Yaji, T. Yamada, M. Yoshino, T. Matsumoto, K. Izui, S. Nishiwaki, Topology optimization in thermal-fluid flow using the lattice Boltzmann method, Journal of Computational Physics 307 (2016) 355-377.

[48] L. Laniewski-Wołłk, J. Rokicki, Adjoint lattice Boltzmann for topology optimization on multi-GPU architecture, Computers \& Mathematics with Applications 71 (3) (2016) $833-848$.

[49] S. Nørgaard, O. Sigmund, B. Lazarov, Topology optimization of unsteady flow problems using the lattice Boltzmann method, Journal of Computational Physics 307 (2016) 291-307.

[50] G. Bird, Molecular Gas Dynamics and the Direct Simulation of Gas Flows, Oxford University Press, 1994.

[51] P. L. Bhatnagar, E. P. Gross, M. Krook, A model for collision processes in gases. I. small amplitude processes in charged and neutral one-component systems, Physical Review 94 (1954) 511-525. 
[52] A. Kawamoto, T. Matsumori, S. Yamasaki, T. Nomura, T. Kondoh, S. Nishiwaki, Heaviside projection based topology optimization by a PDE-filtered scalar function, Structural and Multidisciplinary Optimization 44 (1) (2011) 19-24.

[53] K. Svanberg, The method of moving asymptotes-a new method for structural optimization, International Journal for Numerical Methods in Engineering 24 (2) (1987) 359-373.

54] G. Dimarco, R. Loubere, Towards an ultra efficient kinetic scheme. part i: Basics on the BGK equation, Journal of Computational Physics 255 (2013) 680-698.

[55] L. Wu, J. M. Reese, Y. Zhang, Solving the Boltzmann equation deterministically by the fast spectral method: application to gas microflows, Journal of Fluid Mechanics 746 (2014) 53-84.

[56] H. Sugimoto, Y. Sone, Vacuum pump without a moving part driven by thermal edge flow, in: AIP Conference Proceedings, Vol. 762, AIP, 2005, pp. 168-173. 